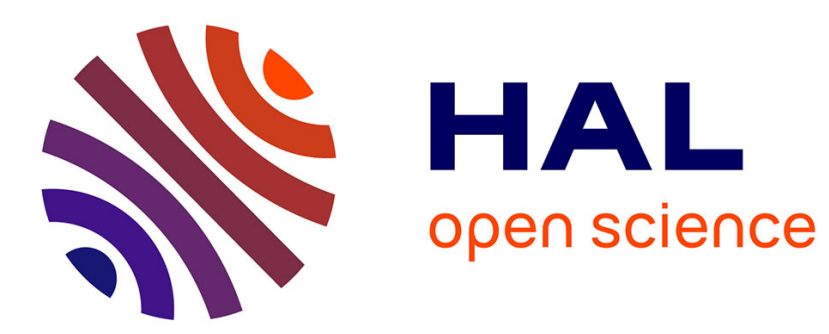

\title{
L'imitation humaine: Une synthèse de 50 années de recherche en psychologie sociale.
}

\author{
Angélique Martin, Nicolas Guéguen, Jacques Fischer-Lokou
}

\section{To cite this version:}

Angélique Martin, Nicolas Guéguen, Jacques Fischer-Lokou. L'imitation humaine: Une synthèse de 50 années de recherche en psychologie sociale.. Canadian Psychology, 2016, 57 (2), pp.101-119. 10.1037/cap0000048 . hal-01999862

\section{HAL Id: hal-01999862 \\ https://hal.univ-rennes2.fr/hal-01999862}

Submitted on 14 Feb 2019

HAL is a multi-disciplinary open access archive for the deposit and dissemination of scientific research documents, whether they are published or not. The documents may come from teaching and research institutions in France or abroad, or from public or private research centers.
L'archive ouverte pluridisciplinaire HAL, est destinée au dépôt et à la diffusion de documents scientifiques de niveau recherche, publiés ou non, émanant des établissements d'enseignement et de recherche français ou étrangers, des laboratoires publics ou privés. 
Martin, A., Guéguen, N. \& Fischer-Lokou, J.

L'imitation humaine : Une synthèse de 50 années de recherche en psychologie sociale 
Human mimicry: Synthesis of 50 years of research in social psychology 


\section{Résumé}

Les premiers travaux sur l'imitation en psychologie sociale ont observé les effets de l'imitation sans s'intéresser aux mécanismes sous-jacents qui la compose et sans en évaluer l'impact social. Depuis, les chercheurs ont manipulé expérimentalement l'imitation, ce qui a permis de mieux comprendre son fonctionnement ainsi que ses effets. La plupart des recherches se sont intéressées aux effets de l'imitation pour la personne imitée et ce n'est que plus récemment que les chercheurs ont mesuré l'impact de l'imitation sur l'imitant. L'ensemble de ces travaux montrent que les individus ont tendance à s'imiter les uns les autres, tant au niveau des comportements non verbaux que verbaux.

Nous nous attacherons ici à mentionner les travaux ayant été menés sur l'imitation, depuis ceux de Tarde (1890) et de Scheflen (1964) aux plus récents. Une synthèse des observations et des résultats obtenus sera exposée et nous relèverons également dans la littérature, quels sont à ce jour les mécanismes connus de l'imitation. Nous ferons état des facteurs pouvant favoriser l'imitation, en augmenter le niveau, ainsi que ceux pouvant à l'inverse l'inhiber. Enfin, nous relèverons les différentes fonctions de l'imitation mises en évidence par les différentes recherches.

Mots Clés : imitation, effet caméléon, interaction sociale, perception sociale, revue de question. 


\begin{abstract}
Early works on mimicry in social psychology analyse its effects through simple observation without addressing its underlying mechanisms or evaluating its social impact. More recently, researchers have experimented with mimicry, giving us a better understanding of its processes and effects. Much of the experimental research focuses on the effects of mimicry on the person who is mimicked and it is only very recently that researchers have measured the impact of mimicry on the mimicker. Current research shows that individuals tend to imitate one another, whether it be on a verbal or non verbal level.

We have reviewed the research conducted on mimicry since the early work of Scheflen (1964) to the most recently published papers. Here we present a synthesis of the observations that have been made concerning mimicry and the results that have been obtained thus far, indicating all the mechanisms that are reported in the literature. We have assessed the factors which may improve mimicry through an increase in level, or in contrast, those that may be able to inhibit mimicry. We have also recorded the different functions of mimicry that have been made clear through recent experimental research.
\end{abstract}

Key words: Mimicry, social interaction, social perception, question review, chameleon effect. 


\section{Introduction}

L'imitation est un processus automatique et non conscient qui regroupe une multitude de comportements que l'on met en place dans un grand nombre de circonstances et dans une grande variété de situations (Chartrand \& Bargh, 1999; Dimberg, 1998; Rizzolatti, Fogassi, \& Gallese, 2001). On a tendance à imiter ce que les gens manifestent, leurs comportements, leurs discours et syntaxes, leurs gestes, leurs mimiques, et leurs postures, aussi bien que leurs états émotionnels et leurs humeurs (Bernieri, 1988; Chartrand \& Bargh, 1999; Dimberg, 1998; Haviland \& Lelwica, 1987; Webb, 1972). Nous imitons le comportement de modèles auxquels nous sommes exposés (médias, famille, amis, ...) mais aussi celui d'étrangers (Chartrand \& Bargh, 1999). Nous imitons même le comportement d'individus n'étant pas présents physiquement comme le rapporte notamment l'expérience de Lakin et Chartrand (2003). Faire ce que les autres font, dire ce que les autres disent et ressentir ce que les autres ressentent semble être une part naturelle de qui nous sommes (Chartrand, Maddux \& Lakin, 2005).

Dans cet article, nous relèverons, dans une première partie, les différents types d'imitation ayant été jusqu'à présent rapportés. Dans une seconde partie, nous mettrons en évidence les mécanismes mis en œuvre dans le processus d'imitation ainsi que ses facteurs inhibiteurs et facilitateurs. Nous ferons ensuite un état des lieux des différents effets de l'imitation tant sur le plan cognitif que comportemental. Enfin, nous relèverons dans la littérature les différentes fonctions de l'imitation.

Dans certaines des expérimentations présentées ci-après, les chercheurs demandaient à des compères d'imiter des sujets naïfs. Pour cela, il est nécessaire de former au préalable les compères à la mise en œuvre expérimentale de l'imitation. De façon générale, lorsque la consigne donnée aux compères est d'imiter les comportements, il faudra que ces derniers reproduisent à l'identique les comportements cibles des sujets à imiter (comme par exemple leurs gestes, postures, mimiques, ...) avec un délai de quelques secondes. Pour ce qui concerne l'imitation verbale, il s'agit, cette fois, de demander aux compères de procéder à une réitération simple des propos des sujets naïfs. Dans ce cas, il faut également préciser que les compères devront veiller à ne pas faire varier la qualité de l'interaction ni sa durée avec les sujets naïfs. Pour cela, les compères ont la consigne de remplacer l'imitation verbale par des mots ou des sons visant à signifier aux sujets naïfs qu'ils les écoutent et les comprennent sans pour autant les imiter. Cela permet de contrôler que la durée des interactions et leur nombre soient sensiblement comparables. Il faut également noter que dans le cadre de ces formations 
à l'imitation, il est précisé aux compères de veiller à ne pas imiter lorsque la situation expérimentale est une condition sans imitation. En effet, comme cela a été souligné, le processus d'imitation est un processus automatique et non conscient, il convient donc de faire un effort pour ne pas imiter et pour respecter les conditions de la situation contrôle. La plupart du temps, la procédure expérimentale ainsi mise en place fait l'objet d'un pré-test. Enfin, pour ce qui est de l'observation de l'imitation, dans la majorité des expérimentations relevées dans la littérature scientifique, la méthode des juges est employée.

\section{Les différents types d'imitation}

En 1890, le juriste, philosophe et sociologue, Gabriel Tarde, a publié une monographie portant sur l'imitation qu'il considérait comme l'élément fondateur du lien social. Néanmoins, ce n'est qu'en 1964 que la première étude expérimentale sur l'imitation est apparue, conduite par le psychiatre Albert Scheflen, qui portait sur la synchronie posturale dans les interactions sociales. Ce dernier s'est intéressé à l'étude de la communication non verbale et notamment à la signification de la congruence posturale dans les interactions. En examinant des vidéos de sessions de psychothérapies familiales, il a constaté que les gens qui avaient des postures semblables à celles d'un autre membre du groupe se montrent plus souvent d'accord avec cette personne et ressentent plus d'empathie envers elle.

\subsection{Imitation verbale}

Faisant suite à ces premiers travaux, des recherches portant sur l'imitation verbale et la synchronie des discours ont été menées. Des nouveau-nés de quatre jours pleurent en réponse aux pleurs d'un autre nouveau-né. Toutefois, ils n'imitent pas ces pleurs s'il s'agit de pleurs synthétiques créés de façon artificielle. Ces résultats montrent que les nouveau-nés sont capables de faire la différence entre des vrais et des «faux » pleurs d'enfants (Simner, 1971). Plusieurs recherches ont également mis en évidence un effet de contagion du rire d'autrui. Young et Frye, (1966) observent qu'une personne à laquelle on fait écouter une blague a tendance à rire davantage lorsque cette dernière est placée dans une situation de groupe (avec des compères qui rient) que lorsqu'elle se trouve seule dans une pièce. Bush, Barr, Mc Hugo et Lanzetta (1989) ainsi que Provine (1992) confirment l'idée selon laquelle les rires ajoutés à une émission télévisée entraînent davantage de rires chez les téléspectateurs. Dans son 
expérience, Provine faisait écouter à des participants (hommes et femmes) une série de 10 rires pré-enregistrés (de 18 secondes chacun). Les résultats ont montré une contagion du rire puisque plus de $90 \%$ des participants rient dès la première écoute. Giles et Powesland (1975) ont relevé l'existence d'une imitation conjointe des accentuations sonores lorsque deux individus conversent ensemble. Une imitation conjointe du timbre de la voix sera par la suite montrée par Neumann et Strack (2000). Cappela et Panalp (1981) observent une synchronie dans le rythme des discours, dans la durée des pauses et la prise de parole entre deux individus conversant ensemble et ce après 20 minutes de conversation. Les travaux de Webb $(1969,1972)$ quant à eux rendent compte d'une imitation conjointe du débit de parole. Les gens imitent les mots, les propositions et syntaxes de phrases entières (Bock, 1989). Levelt et Kelter (1982) observent une imitation du contenu et de la syntaxe d'une question dans la réponse adressée au partenaire d'interaction. Dans une de leur condition expérimentale, les auteurs manipulaient également l'attention des participants et relèvent que l'imitation se produit même lorsque les ressources cognitives des participants sont allouées à la réalisation d'une tâche. Ce qui plaide en faveur du caractère automatique et non-conscient de l'imitation.

\subsection{Imitation des expressions et émotions}

Les recherches font également état d'une imitation importante des comportements non verbaux dans les interactions sociales et notamment de l'imitation des expressions faciales et des émotions. De nombreux travaux ont été menés sur la façon dont les nouveau-nés imitent les expressions faciales des adultes (Kaitz, Meschulach-Sarfaty, Auerbach, \& Eidelman, 1988). Par exemple, les travaux de Meltzoff et Moore (1977) montrent que les enfants d'un mois sourient, tirent leur langue et ouvrent leur bouche lorsqu'ils perçoivent autrui faire de même. Les enfants de 9 mois sont capables d'imiter les émotions exprimées par les expressions faciales de leur mère comme la joie, la tristesse ou la colère (Termine \& Izard, 1988). Dans leur expérience Dimberg, Thunberg et Elmehed (2000) mettent en évidence le caractère automatique de l'imitation. Les auteurs présentaient de manière subliminale (30 millisecondes) des photos de visages heureux, tristes ou neutres aux participants séparés en 3 groupes. Ces derniers étaient ensuite tous exposés de manière consciente à une photo de visage ayant une expression neutre (5 secondes). Les auteurs mesuraient à l'aide d'un électromyographe, l'activité faciale spontanée des participants en réponse à l'exposition aux stimuli présentés de façon subliminale. Bien que les participants n'aient pas perçu le type de visage auquel ils ont été soumis des réponses musculaires différentes ont été enregistrées. 
L'activité de leurs zygomatiques (sourires) est plus importante lorsqu'ils sont exposés à des visages « heureux »; Lorsque les participants ont été exposés de manière subliminale à des visages tristes, c'est le muscle corrugateur (froncement des sourcils) qui présente une activité plus élevée. Les auteurs concluent donc au caractère automatique et non conscient de l'imitation faciale. Les travaux d'Achaibou, Pourtois, Schwartz et Vuilleumier en 2008 viennent confirmer ces résultats. Dans leur recherche, les auteurs faisaient visionner à des participants des vidéos de sport dans lesquels les individus étaient soit heureux, soit en colère. Ils relèvent à l'aide d'un électromyographe une activation musculaire des zygomatiques lorsque les participants sont exposés à des visages heureux. Au contraire, lorsque ces derniers sont exposés à des visages en colère, c'est le corrugateur qui est activé.

Cette activité musculaire traduit bien la capacité que l'on a à mimer les expressions faciales et émotionnelles des individus que nous percevons ou avec lesquels nous interagissons. D'ailleurs les effets de contagion émotionnelle que l'on observe en groupe pourraient provenir des effets de simple imitation des expressions émotionnelles d'autrui. La contagion émotionnelle est définie comme la tendance à imiter les aspects verbaux, comportementaux et psychologiques des expériences/émotions d'une autre personne, ce qui les conduit à ressentir ou exprimer à leur tour les mêmes émotions (Hatfield et al., 1992). Les résultats de l'expérience de Neumann et Strack (2000) montrent qu'un narrateur se montrant d'humeur joyeuse versus d'humeur triste entrainera une humeur congruente chez son auditeur. Ce dernier imite donc l'humeur du narrateur et ce de manière non consciente. L'imitation des expressions faciales, des sons et des postures d'un individu facilite la contagion des émotions de ce même individu (Hatfield, Rapson \& Le, 2011). Dans leurs travaux, Lundquist et Dimberg (1995) constatent que l'imitation de l'expression faciale d'une émotion exposée de façon subliminale entraîne chez les participants le ressenti d'une émotion congruente. Pour Laird, et ses collègues (1994), le fait d'imiter les expressions émotionnelles des autres médiatise nos propres émotions. L'imitation faciale des émotions se produit quelle que soit l'émotion affichée par la source. Hess et Blairy (2001) observent toutefois que la contagion émotionnelle ne se produit qu'en présence de certaines émotions. Ainsi, l'imitation faciale de la joie et de la tristesse entraîne une contagion émotionnelle alors que ce n'est pas le cas avec la colère ou le dégoût. De même, dans leur recherche, Hsee, Hatfield, Carlson et Chemtob (1990) observent que les participants regardant une vidéo dans laquelle une personne raconte une expérience joyeuse ou au contraire une expérience triste ressentent la même humeur après le visionnage. Selon Friedman et Riggio (1981) être simplement assis 
dans une pièce où se trouvent d'autres personnes peut suffire à être « contaminé » par leurs humeurs. Dans leur expérience, ils placèrent trois individus dans une pièce. Deux d'entre eux étaient pré-testés comme étant peu expressifs tandis que le troisième était très expressif. Les résultats montrèrent que les deux participants inexpressifs captent les humeurs du participant expressif et ce même en l'absence de toute communication verbale. Sullins (1991) a trouvé que les personnes très expressives sont davantage capables de transmettre leurs humeurs négatives que les personnes moins expressives. Toutefois, les personnes expressives comme celles non expressives sont en mesure de transmettre aux autres leurs humeurs positives. Enfin, il semble que les individus captent et ressentent davantage les émotions de ceux qu'ils apprécient que les émotions de ceux qu'ils n'apprécient pas (Howard et Gengler, 2001).

Si nos capacités d'imitation participent à créer ces effets de contagion des expressions émotionnelles et des émotions elles-mêmes, on a également montré que lorsque ne peut pas imiter on perturbe le traitement des émotions exprimées par autrui. Stel et van Knippenberg (2008) ont ainsi montré qu'en restreignant la capacité des individus à imiter les expressions émotionnelles de visages présentés sur écran avec une consigne explicite de ne pas le faire et de serrer les dents, les participants mettent plus de temps à identifier ces expressions et commentent plus d'erreurs dans cette identification. De la même manière Rychlowska et al. (2014) ont inhibé la capacité de certains de ses participants à imiter le sourire en leur faisant porter un protège-dents. Les participants devaient tenter de diagnostiquer parmi des visages souriants ceux dont le sourire était sincère ou faux. On observera que l'inhibition de la capacité à sourire a perturbé les performances des individus à distinguer les deux types de sourires.

\subsection{Imitation comportementale}

Les postures du corps et les mouvements des bras font également l'objet d'imitation entre les protagonistes d'une interaction (Bavelas et al., 1987 ; Bernieri, 1988 ; LaFrance, 1979, 1982; LaFrance \& Broadbent, 1976 ; Maurer \& Tindall, 1983 ; Scheflen, 1964). Bernieri (1988) a demandé à des individus de juger plusieurs photos sur lesquelles se trouvaient une mère avec un enfant qui était soit le sien, soit qui était l'enfant d'une autre. Les individus remarquent une plus grande synchronie physique entre une mère et son propre enfant plutôt qu'entre une mère et l'enfant d'une autre. D'autres auteurs observent un effet d'imitation du bâillement (Estow, jamieson \& Yates, 2007 ; Platek, Critton, Myers \& Gallup, 2003). Zajonc, Adelmann, Murphy \& Niedenthal (1987) quant à eux observent une imitation 
des expressions faciales entre les membres de couples lorsqu'ils sont ensemble. Dans leur expérience, ils demandaient à des participants d'évaluer différentes photos de couples. Parmi ces couples figuraient, un couple marié depuis 25 ans, un jeune couple, ainsi qu'un couple de deux personnes ne se connaissant pas et prises au hasard. Les participants ont trouvé plus de similitudes physiques entre les individus mariés depuis 25 ans qu'entre les autres couples. Hsee, Hatfield, Carlson et Chemtob (1990) remarquent que les téléspectateurs imitent les expressions faciales des personnes qu'ils voient à la télévision. Blairy, Herrera et Hess (1999) observent également que les individus imitent les expressions faciales d'autrui. Lorsque les gens perçoivent une personne souffrant d'une blessure, ils ont tendance à mimer la crispation de la bouche du blessé (Bavelas, Black, Lemery \& Mullett, 1986, 1987).

D'autres recherches sur la synchronie entre la parole et les mouvements du corps de deux partenaires en interaction ont été conduites (Bernieri, \& Rosenthal, 1991). Bavelas, Black, Chovil, Lemery \& Mullett (1988) ont mené une expérience dans laquelle une narratrice lit une histoire en classe à des enfants et mime (ou ne mime pas) les gestes effectués par le personnage principal de l'histoire. Dans le cas où la narratrice mime le mouvement de tête décrit par l'histoire, les enfants font de même, imitant le mouvement de la narratrice. Les auteurs ont également montré dans cette expérience que les participants n'imitent pas de façon « rotationnelle » mais en miroir. Dans leur expérience, la narratrice s'inclinait soit sur le côté droit, soit sur le côté gauche et ils mesuraient si en réponse, les participants s'inclinaient à leur tour du même côté ou s'ils s'inclinaient en miroir. Les résultats montrent que les participants imitent les mouvements des individus en miroir.

Les recherches ont également mis en évidence que l'imitation a une forte influence sur les comportements de consommation. Quigley et Collins (1999) ont mené une expérience dans un bar auprès de dyades consommant de l'alcool. Les auteurs observent une synchronie du comportement consistant à porter son verre à sa bouche et à boire. De la même façon, Harakeh, Engels, van Baaren et Scholte (2007) montrent qu'il y a une imitation très forte des comportements tabagiques d'un étranger. Ils observent une synchronie dans la fréquence et l'amplitude des inhalations ainsi que dans la façon de tenir la cigarette ou encore de l'éteindre dans le cendrier même lorsqu'il n'y a pas d'interaction verbale entre les protagonistes. Enfin Tanner, Ferraro, Chartrand, Bettman et van Baaren, (2008) montrent dans leur expérience que les individus ont tendance à imiter les comportements de consommation de leur partenaire d'interaction en choisissant, consommant et préférant l'aliment consommé par ce dernier. 


\section{Les mécanismes en jeu dans l'imitation}

L'être humain est un animal profondément social et ses aptitudes sociales éminemment subtiles aujourd'hui sont le fruit de son évolution. Or, l'humain, plus que tout autre, serait neurologiquement équipé pour mimer autrui. L'existence de véritables «neurones miroirs » (Keysers \& Gazzola, 2010 ; Rizzolatti et Sinigaglia, 2007) qui s'activent automatiquement lorsque l'on observe autrui produire un comportement serait le résultat de cette évolution et pourrait expliquer pourquoi cette activation du mimétisme dans nos échanges sociaux se produirait de manière automatique et non-consciente. Cette spécialisation neurologique justifierait également l'importance des aires cérébrales dévolues aujourd'hui à l'apprentissage dans notre espèce (Meltzoof et Decety, 2003).

\subsection{Le lien entre perception et comportement}

Bargh, Chen et Burrows (1996) suggèrent qu'un des mécanismes en œuvre dans l'imitation serait le lien entre perception et comportement. Selon eux, la simple perception du comportement d'autrui augmenterait la probabilité de produire soi-même ce comportement. On imite les comportements des individus de manière non consciente et cet effet proviendrait d'un lien automatique entre perception et comportement (Chartrand \& Bargh, 1999; Dijksterhuis \& Bargh, 2001 ; Lakin et al., 2003). De nombreuses recherches montrent que le simple fait de penser à des mots ou à des actions entraîne une activité dans les mêmes zones du cerveau que lorsqu'on prononce réellement ces mots ou que l'on réalise ces actions (Decety, Jeannerod, Germain \& Pastene, 1991). La découverte des neurones en miroir plaide en faveur de ce lien direct entre perception et comportement. De nombreuses recherches montrent que le fait de percevoir quelqu'un agir entraîne les mêmes activations neurologiques que si nous accomplissions nous même l'acte (Iacoboni, Woods, Brass, Bekkering, Mazziotta \& Rizzolatti, 1999 ; Gallese, Fadiga, Fogassi \& Rizzolatti, 1996). Le fait de percevoir une cible attraper un objet et le fait d'attraper soi-même cet objet engendre les mêmes réponses musculaires (Fadiga, Fogassi, Pavesi \& Rizzolatti, 1995). Sebanz, Knoblich et Prinz (2003) ont effectué une expérience dans laquelle les participants étaient placés devant un écran qui projetait des images de doigts pointant une direction et qui était entouré d'une bague dont la couleur différait. Les participants avaient pour consigne de dire si la bague était rouge ou verte à l'aide de deux boutons de l'ordinateur (placés un à gauche et l'autre à droite du clavier), les expérimentateurs leur demandaient aussi de ne pas tenir compte de la direction indiquée par le doigt. Les résultats montrent que les participants donnent des réponses plus justes et 
rapides lorsqu'il y a une congruence entre le côté pointé par le doigt et la position de la touche du clavier sur lequel le participant doit appuyer pour donner la bonne réponse. Ainsi, voir quelqu'un faire quelque chose activerait une représentation comportementale correspondante induisant en retour une plus forte probabilité de produire ce comportement. Brass, Bekkering et Prinz (2001) démontrent que le fait de voir un individu bouger un doigt facilite le mouvement du même doigt et gêne le mouvement d'un autre doigt. Berkowitz (1984) montre que le fait de percevoir des actes violents dans les médias active une représentation d'actes violents en mémoire, ce qui entraîne automatiquement d'autres représentations de comportements agressifs et conduit le téléspectateur à se comporter de manière plus violente. Le fait d'être engagé dans un comportement particulier entraîne une meilleure accessibilité en mémoire des traits de catégorie correspondant à ce comportement. Par exemple, venir en aide à une personne, lui rendre service, activerait en mémoire le trait «serviable » ainsi que la représentation comportementale correspondante (Chartrand, Kawada \& Bargh, 2002). Pour Bargh, Chen et Burrows (1996), la perception d'un comportement entraînerait de façon automatique et non-consciente l'imitation de ce comportement du fait de l'activation de stéréotypes qu'elle engendre. En effet, selon ces auteurs, la perception d'un comportement et la réalisation de ce même comportement relèvent de la même structure représentationnelle. D'autres études ont montré cet effet direct de la perception sur le comportement et ont également mis en évidence que cet effet n'est ni intentionnel ni au service d'un objectif perceptible (Chen, Chartrand, Lee-Chai, \& Bargh, 1998).

\subsection{Intention de comprendre}

Pour Over et

Gattis (2010), les processus en œuvre dans l'imitation comportementale ont longtemps été négligés et ne peuvent être expliqués par le lien perception-comportement ni par les neurones en miroirs. Pour ces auteurs, le désir de comprendre les objectifs et intentions des individus avec lesquels nous interagissons est un des mécanismes sur lequel repose l'imitation. D'autres auteurs ont mis en évidence ce lien entre intention de comprendre et imitation comportementale (Bekkering, Wohlschläger \& Gattis, 2000 ; Gattis, 2002). Le lien entre perception et comportement serait médiatisé par les cognitions. L'imitation comportementale n'est pas la copie conforme des comportements des individus imités mais plutôt l'imitation des intentions comportementales de ces individus. L'imitation des mêmes actions par les enfants et les adultes n'est jamais la même du fait de leurs interprétations différentes des intentions et 
des objectifs de la cible. La représentation que se font les individus des comportements ou des intentions comportementales de leurs partenaires d'interaction joue un rôle prépondérant dans l'imitation. Dans leur recherche, Carpenter, Akhtar et Tomasello, (1998) observent que les enfants n'imitent pas les erreurs de leurs partenaires d'interaction. De la même façon, Meltzoff (1995) montre que les enfants n'imitent pas les tentatives comportementales échouées. Enfin, en ce qui concerne l'imitation verbale, de nombreuses recherches plaident en faveur de l'effet médiateur des cognitions entre perception et comportement. Les participants auxquels on fait écouter des phrases dans lesquelles des répétitions grammaticalement fausses sont présentes, n'imitent pas la phrase en l'état mais procèdent à une correction par la suppression des répétitions (Love \& Parker-Robinson, 1972). Dans leur expérience Over et Gattis (2010) montrent que l'imitation verbale s'appuie sur la compréhension des intentions du locuteur. Les auteurs confirment que les participants (des enfants) corrigent automatiquement les erreurs grammaticales lors de leur imitation verbale comme cela avait déjà été suggéré dans de précédentes expériences. L'originalité de leur recherche provient du fait qu'ils observent l'effet de l'imitation verbale d'agents ayant ou n'ayant pas d'intention. Les auteurs observent que les enfants corrigent uniquement les erreurs grammaticales faites par des modèles ayant des intentions (peluche) et ne corrigent pas les phrases prononcées par des modèles sans intention (magnétophone). En effet, lors de la répétition des phrases émises par un modèle ayant l'intention de communiquer, de transmettre un message (matérialisé dans l'expérience par une peluche), les enfants corrigent les erreurs de grammaires. Au contraire, lorsque les enfants n'attribuent pas d'intention de communiquer à la cible, ils répètent les phrases sans en corriger les fautes. Les auteurs concluent donc que la compréhension des intentions de celui qu'on imite est un des mécanismes importants en jeu dans l'imitation, qu'il s'agisse d'imitation comportementale ou verbale.

\subsection{Imitation et désir d'affiliation}

Selon Chartrand et Bargh (1999), un des mécanismes déterminants de l'imitation serait le désir d'affiliation du participant avec la personne avec laquelle il interagit. Pour Baumeister et Leary (1995), les individus ont besoin d'appartenir à des groupes sociaux, ils ont besoin d'attachement et de relations interpersonnelles relativement stables. Ce besoin de rapports sociaux motiverait les comportements des individus lors de leurs interactions sociales. Dans cette perspective, l'imitation pourrait être un outil efficace permettant non seulement la création de liens et de rapports sociaux mais aussi de leur maintien. L'imitation est une forme 
subtile pour démontrer cette volonté d'interaction positive envers l'autre. Des travaux montrent d'ailleurs que les individus présentant des troubles autistiques et qui se caractérisent par des difficultés relationnelles et moins d'empathie à la fois cognitive et affective ont d'ailleurs des difficultés à percevoir et comprendre ces signes subtils traduisant les intentions d'autrui (Mathersul, McDonald \& Rushby, 2013). Van Baaren, Holland et van Knippenberg (2002) ont mis en évidence que l'imitation resserre les liens non seulement entre la personne imitée et la personne imitante mais plus largement entre la personne imitée et les êtres humains. Cette étude suggère que l'imitation pourrait être la cause mais aussi l'effet de l'affiliation. L'imitation se manifeste de manière automatique et non consciente lorsque des liens et des rapports positifs existent entre deux individus mais aussi dans le but de permettre et de maintenir ces liens et rapports. Pour Lakin, Jefferis, Cheng et Chartrand (2003) l'imitation exercerait une influence sur notre comportement et notre jugement à l'égard de l'imitant en raison de l'activation du désir d'affiliation et de rapport social qu'elle produirait auprès de l'individu qui en est la cible. Cette activation se produirait de manière automatique et non-consciente, ne permettant pas, par conséquent, à l'individu de percevoir la raison pour laquelle il agirait ou évaluerait de manière différenciée la personne imitante. Pour ces auteurs, ce caractère automatique et non conscient serait le produit de notre évolution. Chez l'humain, l'interaction sociale est certainement le comportement qui aurait permis la survie et l'adaptation de notre espèce. En effet, l'imitation permet aux individus de réagir rapidement dans des situations de danger, ou encore d'apprendre à se comporter et d'être intégrés dans un groupe afin de bénéficier de sa protection. Le désir d'un individu d'entrer en relation et de créer des liens avec une personne ou un groupe augmente donc son niveau d'imitation. Lakin et Chartrand (2003) ont montré qu'en créant un but d'affiliation non conscient chez un individu, par amorçage avec des concepts en lien avec l'affiliation, on conduit ce dernier à se montrer plus imitant vis-à-vis d'un compère. Pour Lakin et Chartrand (2003), la création d'un but d'affiliation renforcerait l'attention que les individus prêtent à ce qui se passe dans leur environnement social et améliorerait la perception qu'ils ont des comportements de leurs interlocuteurs, ce qui en conséquence, entraînerait davantage d'imitation. Les effets du désir d'affiliation sur l'imitation seraient donc médiatisés par le lien perception-comportement. Parfois ce sentiment d'affiliation produit par l'imitation est si proche qu'il conduit les individus imités à faire part de révélations très personnelles et même intimes. Guéguen, Martin, Meineri et Simon (2013) ont ainsi montré que des étudiantes sur un campus imitées par une enquêtrice et répondant à un questionnaire de sexualité, acceptent plus facilement de 
répondre à des questions très osées sur leur sexualité comparativement à des personnes n'ayant pas été imitées. Pour ces chercheurs, le degré d'affiliation créerait des conditions de confiance telles que cela favoriserait les révélations très personnelles comme nous le ferions avec un ami. Une autre recherche a également montré que l'on donne des informations plus sincères sur les comportements de tris que l'on adopte lorsque l'enquêteur nous imite (Guéguen, 2013). De la même manière, Martin (2010) et Martin, Fischer-Lokou et Guéguen (in press) montrent qu'un enfant du primaire imité par un instructeur lors d'une petite enquête sur ses préférences alimentaires, acceptera plus volontiers de dévoiler des bêtises qu'il a commises dans le passé et de révéler un secret.

\section{Les facteurs renforçant l'imitation}

Il semble qu'il existe des déterminants motivationnels, contextuels et individuels au comportement d'imitation.

\subsection{Rapports et liens entre les individus}

L'imitation serait liée aux rapports sociaux et aux liens existants entre les individus qui interagissent. Les sentiments et les liens que l'on a envers une personne semblent augmenter le niveau d'imitation. On imite davantage une personne qu'on apprécie (Chartrand et Jefferis, 2003) qu'une personne qu'on n'apprécie pas (Stel, Bergmann, Jansen, Muller \& Wagenaar, 2005). On sait également que le niveau d'imitation (et notamment d'imitation comportementale) est un indicateur des rapports existants dans le groupe (Chartrand et al., 2005). Suite aux premiers travaux, les chercheurs ont remarqué que l'imitation était un indicateur des affinités et des liens entre les individus (Scheflen, 1964 ; Bavelas et al., 1986 ; LaFrance \& Broadbent, 1976). De ce fait, si les affinités entre les partenaires augmentent, alors les individus devraient davantage adopter les postures et les comportements de l'autre. Les recherches sur l'imitation entre les mères et les enfants pourraient ainsi être réinterprétées. En effet, il y a beaucoup d'amour entre une mère et son enfant et l'existence de liens très fort entre l'un et l'autre pourraient expliquer qu'il y ait entre eux une très forte imitation (Bernieri, 1988 ; Meltzoff \& Moore, 1977 ; Termine et Izard, 1988). Cela pourrait également nous aider à comprendre pourquoi les couples mariés depuis longtemps, et qui ont donc un niveau d'affinité très élevé, ont tendance à se ressembler (Zajonc et al., 1987). Le mimétisme des postures améliorerait donc les rapports entre les individus et augmenterait les affinités (LaFrance 1979). Toutefois, les affinités et liens augmentent également le mimétisme 
des postures (LaFrance \& Broadbent 1976 ; Scheflen, 1964). Une recherche a montré un lien entre l'imitation et l'intimité ressentie entre les individus (Ashton-James et al., 2007). Les chercheurs observent que les participants sont plus imitants lorsqu'on les centre au préalable sur des problématiques personnelles ou lorsqu'ils perçoivent des informations personnelles sur autrui (Jefferis, van Baaren \& Chartrand, 2009). Le fait de partager avec autrui des informations personnelles renforcent les liens et relations qui les unissent, ce qui entraîne en retour un niveau d'imitation plus élevé entre les partenaires d'interaction.

Enfin, une recherche de Guéguen, Martin et Vion (2009) montre que l'induction d'un sentiment de familiarité entre deux individus augmente le niveau d'imitation. Il semble également que la similarité perçue, entre deux individus, a un impact plus fort, sur leur imitation réciproque, lorsqu'elle est en lien avec l'identité de soi. (Guéguen \& Martin, 2009)

\subsection{Désir d'affiliation}

Le désir d'affiliation semble également pouvoir être un facteur motivant l'imitation. En effet, lorsque l'on introduit un but d'affiliation conscient ou non conscient, les individus se montrent davantage imitants (Lakin \& Chartrand, 2003). Dans leur expérience, ces chercheurs informaient des participants qu'ils allaient observer par vidéo une personne qu'ils rencontreraient quelques minutes après. En condition contrôle, cette information n'était pas donnée. Sur la vidéo, la personne se grattait plusieurs fois le visage durant la séquence, les auteurs mesuraient la fréquence de réplication de ce comportement chez le participant. Les résultats ont révélé qu'un but d'affiliation anticipé a induit une imitation plus importante de la cible. Une seconde expérience utilisant une tâche d'amorçage a montré les mêmes effets. Lors d'une tâche de reconnaissance de mots, les participants étaient ou non exposés à des mots en lien avec l'affiliation et le rapport social (amis, partenaires, ensemble...). Les chercheurs observent que les participants préalablement soumis à ces mots, et seulement à ces derniers, ont manifesté ultérieurement plus d'imitation du comportement de la cible présentée sur vidéo.

\section{a) Exclusion sociale}

Dans une troisième expérience, Lakin et Chartrand (2003) ont montré que la réussite ou l'échec d'une interaction sociale initiale pouvait prédire le niveau d'imitation ultérieur d'une personne. Ils ont demandé à un compère, lors d'une première interaction en dyade, de se montrer amical (réussite interaction) ou non amical (échec interaction) avec un participant. 
Par la suite, sous prétexte d'une autre expérience, le participant devait regarder une cible sur vidéo qui se grattait plusieurs fois le visage. Les résultats ont montré que, lorsqu'il y a eu échec préalable de l'interaction, le taux d'imitation a été plus important. Les auteurs concluent que l'échec d'une interaction préalable activerait le désir de réussir une interaction future ce qui engendrerait en retour une imitation plus prononcée afin d'exprimer à autrui ce désir. Pour les auteurs, de tels résultats plaident en faveur du lien existant entre imitation et désir d'affiliation. Lakin, Chartrand et Arkin (2008) montrent également que l'exclusion favorise l'imitation mais cela s'observe uniquement auprès d'une personne du groupe d'appartenance du participant. L'imitation serait donc un processus permettant aux individus de rester intégrés à leur groupe d'appartenance ou de le réintégrer en cas d'exclusion.

\section{b) Imitation et culpabilité}

Martin, Guéguen et Fischer-Lokou (2010) ont montré que l'éveil d'un sentiment de culpabilité chez un individu augmente son degré d'imitation à l'égard d'un autre partenaire d'interaction. Dans l'expérience un compère avait pour consigne de bousculer le participant en sortant d'un bureau entraînant la chute de la pile de copies que le compère portait. Dans la condition expérimentale, le compère culpabilisait le participant en lui laissant entendre qu'il était responsable du fait de son inattention. Il ajoutait que désormais, à cause de lui, il était très embêté puis il partait. Dans la condition contrôle, au contraire, le compère s'excusait et endossait l'entière responsabilité de la bousculade à l'origine de la chute de ses documents. Le participant était ensuite placé devant un écran et devait juger une jeune femme présentée sur une vidéo dans le cadre d'une prétendue étude sur la gestion des impressions. Les résultats montrent que les participants sont d'autant plus imitant qu'on a préalablement éveillé chez eux un sentiment de culpabilité. Il semble que l'exclusion sociale engendrée par la culpabilité ait induit une imitation plus forte de la part du participant, ce qui plaide en faveur du fait que l'imitation soit un facteur d'affiliation et d'intégration sociale.

\section{c) Sentiment de différence vis-à-vis des autres}

Selon la théorie de la différenciation optimale de Brewer (1991), les individus tendent à trouver un équilibre entre leur désir de se distinguer des autres (se sentir unique et différent) et leur désir de ressemblance aux autres. Lorsque les individus se sentent trop différents ou trop semblables, ils seraient motivés pour rééquilibrer la balance. Uldall, Hall et Chartrand (2008) ont fait compléter un test de personnalité à des participants (des étudiants). Suite à ce 
test, les chercheurs disaient aux participants qu'ils avaient une personnalité type, très similaires à la plupart des étudiants, ou au contraire ils leur disaient qu'ils avaient une personnalité atypique, extrêmement inhabituelle dans cette université. Les étudiants devaient ensuite interagir avec un autre étudiant de l'université (en réalité un compère). Les résultats montrent que les individus pensant avoir la même personnalité que la plupart des étudiants imitent moins leur partenaire d'interaction que ceux qui pensent être très atypiques. Il semble donc que les individus imitent davantage les autres lorsqu'ils se sentent trop différents des membres de leur groupe d'appartenance.

\section{d) Stéréotypes}

Les individus partageant un stéréotype donné sont davantage imités que les individus remettant en question ce stéréotype. Pour Castelli, Pavan, Ferrari et Kashima (2009), le fait de partager un stéréotype avec une personne revient à partager des opinions, attitudes, croyances avec cette personne. Ce partage d'opinion, cette conformité au stéréotype renforce donc les liens qui existent entre les individus ainsi que leur désir d'établir une relation en éveillant un sentiment de similarité, de ressemblance entre les individus. Lorsque les individus partagent une opinion, une idée, un stéréotype, ils semblent plus proches des autres participants ayant les mêmes idées, opinions ou stéréotypes et donc sont davantage imités. Au contraire, un individu ne partageant pas les mêmes opinions que le participant est perçu comme différent et est donc moins imité. Cet effet du partage ou du non partage des stéréotypes sur l'imitation se produirait de manière automatique et non consciente dans le but de signifier implicitement à l'autre un désir d'affiliation, de rapports sociaux avec lui. Dans leur recherche, Castelli et al. (2009) demandaient à des compères interagissant verbalement avec les participants de leur parler des personnes âgées soit en partageant les stéréotypes liés à la vieillesse, soit en ne les partageant pas. De la même façon, tout au long de l'interaction, les compères avaient pour consigne de bouger leur jambe ou de se caresser la joue. Les chercheurs observent une plus forte imitation des compères parlant des stéréotypes propres à la vieillesse plutôt que des contre-stéréotypes. Les participants se touchent également significativement plus la joue et bougent plus le pied (plus d'imitation) dans la condition où le compère utilise des traits stéréotypiques pour décrire la vieillesse.

\subsection{Conception de soi ${ }^{1}$ interdépendante}

\footnotetext{
${ }^{1}$ Traduit de l'anglais « self construal » qui correspond à la conception de soi en tant que construction sociale.
} 
Les individus ayant une conception de soi indépendante ont tendance à se concevoir comme des individus autonomes, distincts des autres et se décrivent essentiellement avec des traits personnels uniques. Au contraire, les individus ayant une conception de soi interdépendante ont tendance à se définir comme appartenant à un groupe et entretiennent des relations avec les autres (Cross \& Madson, 1997). Ces derniers désirent davantage ressembler aux membres de leur groupe et sont donc plus imitants que ceux ayant une conception de soi indépendante qui cherchent davantage à se distinguer des autres (van Baaren, Maddux, Chartrand, De Bouter \& van Knippenberg, 2003). Dans une autre étude, ces mêmes auteurs ont observé que les Japonais qui ont une culture collectiviste et possèdent une conception de soi interdépendant se montrent plus imitants que les américains qui évoluent dans une culture encourageant l'individualisme et favorisant une conception de soi indépendant. Van Baaren et al. (2003) ont montré que les individus ayant préalablement été soumis à une tâche d'amorçage contenant des mots relatifs à l'interdépendance se montrent, dans la seconde partie de l'expérience, plus imitants que les autres. Au contraire, ils constatent que les individus ayant été préalablement soumis à un amorçage avec des mots en lien avec l'indépendance se montrent moins imitants que le groupe contrôle.

\subsection{Monitorage de soi et dépendance au contexte}

Cheng et Chartrand (2003) rapportent que les personnes ayant un monitorage de soi (contrôle de soi) fort sont plus attentives aux signaux de contexte et se montrent donc plus imitantes notamment à l'égard des personnes d'un groupe supposées proches d'elles ou envers lesquelles elles croient (faussement) qu'elles ont plus de pouvoir. Dans leur recherche, van Baaren, Horgan, Chartrand et Dijkmans (2004) ont montré que moins les individus ont un score élevé au Embedded Figure Test (mesurant le degré de dépendance au contexte) plus leur score d'imitation est élevé. Dans un premier temps, les auteurs faisaient remplir le test (EFT) aux participants afin de déterminer leur dépendance/indépendance au contexte. Ils leur demandaient ensuite de regarder une vidéo d'une durée de 8 minutes sur laquelle une femme exécutait un certain nombre de tâches. Les chercheurs relèvent des corrélations négatives entre le score obtenu au Embedded Figure Test et l'imitation des comportements de la femme présentée sur la vidéo. Les participants se touchent significativement plus le visage et bougent 
davantage les lèvres et les pieds lorsque leur score à l'EFT est bas, c'est-à-dire lorsqu'ils sont fortement dépendants au contexte.

\subsection{Statut}

Le niveau d'imitation varie également en fonction du statut du protagoniste. Ainsi, Moore (1995) observe une imitation plus importante par les pairs de la personne manifestement dominante du groupe (la plus regardée, en position centrale). De la même façon, Hsee, Hatfield, Carlson et Chemtob (1990) montrent que les individus ont tendance à imiter et à « capter » davantage les émotions d'autres individus puissants, ayant du pouvoir et donc un statut important dans le groupe. Ashton-James et Levordashka, (2013) observent également que l'on imite plus une personne de haut statut mais cela s'observe chez des personnes ayant un niveau de narcissisme élevé. Ces dernières auraient un plus fort désir d'être appréciées d'autrui mais cela ne serait intéressant que si la personne susceptible de les juger est de hautstatut. Avec un pair ou une personne de plus bas statut, ce désir narcissique ne pourrait être satisfait.

\subsection{Empathie}

L'empathie est également un facteur dispositionnel facilitateur de l'imitation non consciente. Dans sa définition de l'empathie, Lipps (1903) observe que l'imitation des comportements des autres joue un rôle essentiel dans la genèse de l'empathie. Puisqu'il existe un lien étroit entre l'imitation, les affinités et les relations entre individus, l'empathie a également une influence sur l'imitation des comportements d'un partenaire avec lequel on interagit. Chartrand et Bargh (1999) ont montré que les individus avec un haut niveau d'empathie (mesuré à l'aide de l'échelle de Davis, 1983) sont davantage imitant. Les travaux de Bavelas, Black, Lemery, et Mullett, (1987) montrent également un lien entre l'empathie ressentie à l'égard d'un individu et le niveau d'imitation. Selon les auteurs, une personne avec un haut niveau d'empathie se montre plus attentive aux comportements des autres ce qui entraîne en retour une plus forte imitation. Pour Carr, Iacoboni, Dubeau, Mazziotta et Lenzi (2003) l'imitation correspond à la traduction d'une certaine empathie à l'égard de la personne imitée. Une personne empathique a tendance à imiter davantage les comportements, les postures et les expressions faciales des individus avec lesquels ils interagissent qu'une 
personne n'étant pas empathique. Dimber, Andreasson et Thunberg (2011) ont mesuré l'activité par électromyographie (EMG) des muscles zygomatique et corrugateur du visage en réaction à la perception de photographies de visages présentant des émotions positives ou négatives. Ils observeront une plus forte activité EMG, signe du degré d'imitation, auprès de personnes diagnostiquées préalablement comme très emphatiques. De la même manière, Sonnby-Borgström (2002) a montré que la nature des sentiments exprimés par des individus exposés à des émotions de personnes est corrélée avec leur niveau d'imitation et que des sujets faiblement empathiques pouvaient produire des effets contre-imitatifs selon les émotions (par exemple sourire face à une expression de colère).

\subsection{Humeur}

Van Baaren, Fockenberg, Holland, Jansen et van Knippenberg (2006) montrent que l'humeur affecte les comportements d'imitation. Une humeur positive facilite les comportements automatiques et spontanés alors qu'une humeur négative entraîne davantage de comportements réfléchis et conscients. L'humeur positive permet à l'individu de percevoir son environnement comme étant non menaçant ce qui entraîne un traitement des informations contextuelles. De ce fait, une humeur positive induit automatiquement la prise en compte du comportement d'autrui et par conséquent un niveau d'imitation plus élevé de ce dernier.

\section{Les facteurs inhibant l'imitation}

\subsection{Contagion émotionnelle et Exo-groupe}

Pour Chartrand, Maddux et Lakin (2005), il existe des limites à la «contagion émotionnelle ", on ne capte pas toutes les émotions des personnes que l'on croise, certaines circonstances peuvent inhiber une telle imitation. Dans leur expérience, les auteurs faisaient visionner à des républicains une conférence de presse donnée par l'ancien président Ronald Reagan dans laquelle ce dernier exprime clairement diverses émotions telles que la colère ou la joie. Conformément aux attentes des auteurs, les républicains rapportent, au fur et à mesure du visionnage, les mêmes émotions que celles exprimées par Reagan. En revanche, au fur et à mesure de la conférence, les démocrates rapportent uniquement des sentiments négatifs. Il semble donc que l'on capte les émotions de nos amis, des membres de notre famille, de nos 
compagnons ou même d'étrangers mais pas celles de nos opposants.

\subsection{Attractivité du modèle}

Selon Parks (1980), l'imitation peut varier en fonction de la qualité de l'interaction et donc de l'attractivité du modèle. Les individus ont tendance à moins imiter une cible appartenant à un exo-groupe, ce qui semble aller dans le sens d'un lien entre appréciation et imitation (Yabar, Johnston, Miles \& Peace, 2006). On imite moins les individus avec lesquels on désire moins entrer en relation, on imite moins les individus qu'on n'apprécie pas (Stel, Blascovich, Mc Call, Mastop, van Baaren \& Vonk, 2009). La tendance automatique et nonconsciente d'imiter les individus qui nous entourent peut ainsi être annulée par des émotions négatives.

\subsection{Imitation et stigmas sociaux}

La recherche de Johnston (2002) se concentre sur la personne imitée plutôt que sur celle qui imite. Nous savons depuis les travaux de Lott et Lott (1961) que certaines personnes sont plus imitées que d'autres. Johnston a examiné si la stigmatisation d'un individu pouvait affecter le niveau d'imitation des individus. Dans sa première expérience, Johnston demandait aux participants de goûter des crèmes glacées de différents parfums et de noter leurs préférences à l'aide d'un questionnaire. Les participants étaient en présence d'un compère de l'expérimentateur qui avait pour consigne de consommer soit une grosse quantité soit une petite quantité de crème glacée. Johnston a également fait varier les compères, dans une condition le compère présentait un stigma, il était obèse, dans l'autre condition il ne l'était pas. Elle mesurait ensuite la consommation de crèmes glacées des participants. Les résultats montrent que, face à une personne obèse, un participant inhibe le comportement d'imitation. Dans une seconde expérience, le stigma possédé par le compère était une marque de naissance sur le visage. Dans cette seconde expérience, le stigma n'affecte pas le comportement d'imitation des participants, lesquels consomment autant de glace que le compère quelle que soit la condition (peu de glace ou autant de glace). Les stigmas sociaux semblent être un facteur inhibant le niveau d'imitation mais seulement lorsque le coût de l'imitation est associé au stigma. Le fait que l'individu cible soit stigmatisé peut inhiber l'imitation suite aux influences normatives ou informationnelles du stigma. L'imitation d'un individu stigmatisé 
peut mener à un stigma-par-association négatif (Neuberg, Smith, Hoffman, \& Russell, 1994).

\subsection{Imitation et conception de soi}

Comme nous l'avons vu précédemment, les participants ayant une conception de soi indépendante se montrent moins imitants que ceux ayant une conception de soi interdépendante. Il semble même que l'indépendance puisse annuler les effets de l'imitation. En effet, dans leur expérience, van Baaren \& al. (2003) remarquent que les participants ayant au préalable été amorcés avec des mots en lien avec l'indépendance se montrent dans ce cas moins imitants que les participants du groupe contrôle.

\section{Les effets de l'imitation}

Jusqu'à ce jour, aucune recherche n'a mis en évidence l'existence de différences entre l'imitation verbale et l'imitation gestuelle. Ainsi, van Baaren et al. (2003) montrent que l'imitation verbale influence les comportements altruistes à l'égard de la personne qui nous imite, tout comme l'imitation des comportements non verbaux (van Baaren et al., 2004).

D'autres recherches ont montré qu'il n'existe pas de différence entre les hommes et les femmes concernant l'imitation et son impact (Ashton-James et al., 2007 ; van Baaren et al., 2004). Une étude a néanmoins relevé que l'imitation avantageait davantage les femmes que les hommes en matière de reconnaissance des émotions à partir des expressions faciales (Stel \& van Knippenberg, 2008). Ces chercheurs ont en effet demandé à leurs participants, des hommes et des femmes, de regarder des photographies présentant des visages exprimant différentes expressions émotionnelles (peur, surprise, joie...). Une condition de contrôle de l'imitation de l'expression était introduite dans laquelle on demandait aux participants d'éviter de répliquer les expressions du visage et de conserver les dents serrées. La tâche consistait à reconnaître le plus rapidement possible l'expression faciale présentée. Le temps de réaction était mesuré ainsi que l'erreur/non erreur du décodage de l'expression. Les résultats montreront que chez les femmes seulement le contrôle de l'imitation faciale a augmenté le temps de réaction des participantes et a également augmenté leur taux d'erreur à décoder l'expression exprimée par la cible. Pour les chercheurs, conformément à la théorie de la cognition incarnée des émotions (Barsalou, Niedenthal, Barbey, \& Ruppert, 2003), l'imitation favoriserait la reconnaissance des émotions car, dans cette condition, la personne prendrait la route la plus rapide qui se fonde sur l'empathie émotionnelle qui pourrait servir d'indice à la 
reconnaissance comparative plutôt qu'une route longue qui implique que l'émotion de décode en confrontant l'émotion perçue et un registre de connaissance sur les émotions stockées en mémoire. Or, pour Stell et van Knippenberg (2008) si la restriction de l'imitation a bien perturbé le décodage des émotions uniquement chez les femmes, ce serait parce qu'elles auraient plus d'empathie que les hommes. Sur plan neurologique, une recherche récente de Korb et al. (2015) montre d'ailleurs que chez des femmes seulement la répétition d'une stimulation magnétique transcranienne (rTMS) appliqués au cortex moteur primaire droit et au cortex somatosensoriel primaire droit réduisait le niveau d'imitation des expressions faciales. Si ce lien entre genre et effet de l'imitation est bien mis en évidence, il faut se garder de généraliser. Lorsque les hommes et les femmes bénéficient d'un temps suffisant (présentation du stimulus suffisamment longtemps) pour accéder aux émotions de l'individu on ne trouve pas de différence de genre (Stel, van Baaren \& Vonk, 2008). On notera également qu'une recherche récente de Kulesza et al. (2015) reprenant sensiblement la même méthodologie que Stell et van Knippenberg (2008) ne met pas en évidence de différence de genre.

De manière générale, malgré certaines recherches montrant parfois des différences de genre, on constate le plus souvent que les effets de l'imitation s'observent de manière assez constante tant sur le plan cognitif : (attrait de la personne, appréciation de l'interaction, Chartrand et Bargh, 1999; perception de l'autre comme étant plus empathique, Maurer et Tindall, 1983) que sur le plan comportemental (l'imitation augmenterait notamment l'aide à autrui, van Baaren et al., 2002, 2004).

\subsection{Sur le plan cognitif}

a) Création, maintien de relations et impact sur le jugement social

Dans une recherche longitudinale impliquant l'observation entre lycéens et enseignants, LaFrance (1979) a observé que le degré de synchronie des comportements nonverbaux et des expressions verbales dans la dyade enseignant-élève est un prédicteur de la qualité du rapport existant entre les deux personnes. Dans une seconde recherche, LaFrance (1982) a montré que les étudiants ont tendance à imiter la posture de leur professeur et que le niveau d'imitation de ce dernier est positivement corrélé aux rapports existants entre celui-ci et les étudiants. L'imitation a un effet sur le jugement social ; on apprécie davantage une personne qui nous imite (Chartrand \& Bargh, 1999 ; Chartrand \& Jefferis, 2003), on perçoit 
les personnes qui nous imitent comme plus amicales (Bavelas \& al., 1987), on trouve l'interaction plus agréable lorsque l'on est imité (Chartrand \& Bargh, 1999) et également lorsque l'on imite (Stel \& Vonk, 2010) on apprécie davantage un agent virtuel délivrant un message persuasif et on trouve le message plus convaincant (Bailenson \& Yee, 2005). L'imitation conduit les individus à se percevoir comme étant plus proches (van Baaren et al., 2003 ; Ashton-James et al., 2007). L'imitation augmente le sentiment d'affiliation (Lakin \& Chartrand, 2003) tandis que l'absence d'imitation interpersonnelle entraîne l'exclusion sociale (Lakin et al. 2008). Dabbs (1969) a trouvé que les participants évaluent un compère qui les imite comme ayant de bonnes idées et ayant bien informé. Il remarque également que pratiquer «l'anti-imitation » c'est-à-dire faire l'inverse de ce que quelqu'un fait, ou manifester des émotions contraires à celle exprimées par la personne avec laquelle on interagit diminue l'attrait de « l'anti-imitant » par rapport à celui qui ne fait rien ou qui se montre imitant.

b) Persuasion

Dans la recherche de Bailenson et Yee (2005) l'agent virtuel délivrant un message de prévention est perçu comme étant plus persuasif lorsqu'il se montre imitant. L'imitation a des conséquences sur les comportements prosociaux et entraîne une meilleure appréciation des interlocuteurs. Selon Cialdini (2004), les individus sont d'autant plus sensibles à la persuasion lorsqu'elle est mise en œuvre par une personne qu'ils apprécient, qu'ils croient et dont ils se sentent proches. Dans la mesure où l'imitation entraîne ces sentiments, il n'est pas étonnant qu'elle soit à l'origine de plus de succès lors des tentatives de persuasion. D'autres recherches montrent que l'imitation peut également influencer les décisions d'achats. Dans l'expérience de Guéguen (2005), des vendeurs de produits technologiques (lecteurs MP3 et vidéo) avaient pour instruction d'imiter les propos et les gestes de la moitié des clients qui les sollicitaient pour une information, un conseil sur un choix de modèle. Les résultats ont montré que lorsque les clients ont été imités, ils évaluent d'une part le vendeur comme étant plus sympathique, plus à l'écoute et plus professionnel, d'autre part ils évaluent plus positivement le magasin de manière plus générale. Van Swol (2003) remarque que les individus évaluent leur partenaire d'interaction comme étant plus persuasif lorsque ce dernier est imitant. L'auteur n'observe toutefois aucun effet de l'imitation sur le changement d'attitude et d'opinion dans le sens de celles affichées par l'imitant (Van Swol, 2003 ; Van swol \& Drury, manuscrit en progression).

c) Séduction 
Selon Guéguen, (2009) et Guéguen et Martin (2008), l'imitation augmente l'attrait des individus à l'égard de la personne imitante dans un contexte de séduction. Lors de session de speed-dating, une jeune femme avait pour consigne d'imiter un individu sur deux. A l'issue de la session, les chercheurs mesuraient le désir manifesté par les participants de revoir la jeune femme (classement) ainsi que l'évaluation de l'interaction sociale et de l'attirance éprouvée pour la complice de l'expérimentateur. Les résultats montrent qu'une jeune femme qui imite un homme accroît la désirabilité que ce dernier a de la revoir. L'imitation dans ce contexte a également un impact sur l'évaluation de l'interaction ainsi que sur l'attirance que le participant attribue à la jeune femme.

\section{d) Négociation}

Dans une situation de négociation, l'imitation faciliterait la coordination interpersonnelle en ouvrant les canaux de communication (Fischer-lokou, Guéguen, Lamy, Martin, \& Bulllock, 2014). Maddux, Mullen, \& Galinsky, 2008). Ainsi, les négociateurs utilisant une stratégie d'imitation obtiendraient davantage d'informations de l'autre partie en les plaçant dans un climat de confiance, ce qui aurait également pour conséquence d'augmenter la coopération. En somme, l'imitation dans un contexte de négociation augmente la probabilité pour les deux parties de trouver des solutions et des accords plus pertinents.

\section{e) Création}

Dans de récents travaux, Ashton-James et Chartrand (2009) ont montré que l'imitation pouvait également influencer le type de traitement cognitif des individus. Selon ces auteurs, l'imitation serait un signal social permettant la pensée créative. Les travaux de Guilford (1959) ont démontré que la pensée convergente et la pensée divergente entraînent la créativité. Dans leur étude, Ashton-James et Chartrand (2009) montrent que l'imitation à un impact sur le style cognitif mis en place par les individus. Elles observent que l'imitation est perçue comme un signe de coopération ce qui entraîne la pensée convergente. Au contraire, la non imitation est un signal d'innovation et d'improvisation et entraîne la pensée divergente.

\section{f) Auto-régulation}

Dalton, Chartrand, et Finkel (2010) ont examiné l'effet de l'imitation sur l'autorégulation et ont remarqué qu'une coordination sociale élevée augmente la mobilisation des ressources cognitives. La capacité d'auto-régulation est, elle aussi, améliorée entrainant en 
retour un contrôle de soi plus élevé et enfin davantage d'habileté à accomplir ses propres actions. Les auteurs constatent ainsi que l'imitation entraîne la perception d'une coordination sociale élevée et donc une meilleure régulation sociale. Dans leur recherche, les participants ayant préalablement été imités réalisent un meilleur score à la tâche d'auto-régulation, ils déclarent également manger moins de mauvaise nourriture et procrastinent moins que les participants non imités. Kouzakova (2009) constate également que l'imitation entraîne de meilleures capacités cognitives chez l'individu imité. L'auteur montre en effet que les participants imités ont de meilleures performances à la tâche du Stroop que les participants non-imités (lesquels d'ailleurs réussissent moins bien que le groupe contrôle). Les résultats montrent également que les participants imités ont une meilleure fluidité verbale (meilleur accès au vocabulaire en mémoire) mais qu'ils ont une plus mauvaise capacité de mémorisation des expressions émotionnelles faciales. Martin (2010) et Martin et Guéguen (2015) remarquent également que l'imitation verbale entraîne chez les enfants une plus grande attention prêtée au document présenté par l'expérimentateur imitant (un texte illustré). L'auteur remarque également que les enfants imités retiennent plus d'informations contenues par ce document et font moins d'erreur de restitution que les enfants n'ayant pas été imités.

\section{g) Conception de soi}

Les individus ayant préalablement été imités ont tendance à se percevoir par la suite comme étant plus interdépendants. Dans leur expérience, Ashton-James, van Baaren, Chartrand, Decety et Karremans (2007) observent qu'à l'issue d'une interaction durant laquelle ils ont été imités, les individus s'évaluent davantage orientés vers les autres (plus interdépendants) que les individus n'ayant pas été imités. Guéguen (2013) constate que des individus imités manifestent plus de conscience de soi publique et privée que des personnes no-imitées. Ce chercheur observe également que l'anxiété sociale diminue après qu'une personne ait été imitée. De la même façon, l'imitation entraîne des effets chez les personnes imitantes. Ces dernières se perçoivent plus en relation avec les autres et ont donc une conception de soi plus interdépendante lorsqu'elles ont au préalable imité leur partenaire d'interaction que lorsqu'elles ne l'ont pas imité (Redeker, Stel \& Mastop, 2011). Pour ces auteurs, l'imitation peut être reliée à l'interdépendance car toutes deux ont un effet sur l'augmentation de l'affiliation et favorisent les comportements pro-sociaux.

\section{h) L'imitation empêche de déceler le mensonge}


Jusqu'ici les travaux ont montré des effets positifs de l'imitation. Néanmoins, dans leur recherche, Stel, van Dijk et Olivier (2009) ont souligné une conséquence négative de l'imitation. Il semble que celle-ci empêche les gens de déceler si la personne avec laquelle on interagit, et donc qu'on imite (inconsciemment ou parce qu'on nous l'a demandé), nous ment ou nous dit la vérité. En effet, il semble que le fait de ne pas imiter l'autre conduit à davantage se rendre compte qu'il nous ment. Au contraire, l'imitation consciente ou non consciente d'un individu qui nous ment, nous empêche de capter les signaux qui devraient nous avertir de la supercherie.

i) Estime de soi

L'imitation a également un impact sur l'estime de soi des individus imités. En effet, Kouzakova, Karremans, van Baaren et van Knippenberg (2010) ont observé qu'après avoir été imités, les individus ont une meilleure estime d'eux-mêmes. Concernant les individus qui n'ont pas été imités, et dont l'estime de soi a diminué, les auteurs remarquent des différences dans l'évaluation de leurs relations personnelles (amitiés, relations amoureuses). Le fait de ne pas être imité est inhabituel et engendre un sentiment d'exclusion sociale qui conduit à une diminution de l'estime de soi, en réponse à cela, les individus évaluent plus favorablement leurs relations personnelles, de sorte à se sentir mieux et à restaurer leur estime de soi.

\section{j) Stéréotypes}

D'autres recherches ont montré un impact négatif de l'imitation concernant le fait de se conformer davantage aux stéréotypes sociaux existants. Dans leur expérience, Leander, Chartrand, et Wood (2011) ont fait passer des tests de mathématiques à des filles et des hommes (expérimentation 1), à des américains d'origine asiatique ou caucasienne (expérimentation 2). Les résultats montrent que les participants ayant été imités et faisant partie de la population dont le stéréotype social leur attribue des aptitudes et des compétences moindres en mathématiques réussissent moins bien le test. En d'autres termes, les participants imités se conforment davantage aux stéréotypes de genres et d'origines ethniques que les participants non imités. Selon les auteurs, cet effet est dû au fait que les participants imités prêtent davantage d'attention au contexte, ont une conception de soi plus interdépendante et ont un plus grand désir d'affiliation. L'ensemble de ces facteurs rendent les individus imités plus sensibles et plus liés aux signaux de leur environnement social, ce qui entraîne en retour un plus fort conformisme aux stéréotypes. Si le fait d'être imité renforce certains stéréotypes, 
la recherche montre toutefois que le fait d'imiter peut changer certains stéréotypes. Inzlicht, Gutsell et Legault (2012) observent que des non noirs qui devaient observer sur vidéo un groupe essentiellement composé de noirs stigmatisent moins ces derniers lorsqu'ils ont eu pour instruction d'imiter le comportement des acteurs.

\subsection{Sur le plan comportemental}

a) Comportements prosociaux

Van Baaren, Holland, Steenaert, et van Knippenberg (2003) ainsi que jacob et Guéguen (2013) observent qu'un client donne davantage de pourboire à une serveuse qui l'a imité verbalement lors de la prise de sa commande. Les auteurs concluent que l'imitation accroît la préférence et le sentiment de proximité envers la serveuse qui les a imités et ceci vient accroître leur générosité.

Il y a un effet de l'imitation sur le comportement d'aide spontanée (aide sans sollicitation) dans le sens où les personnes qui ont été auparavant imitées vont se montrer plus aidantes à l'égard de l'imitant. Il semblerait que l'imitation favorise les comportements altruistes. Dans l'expérience de van Baaren, Holland, Kawakami et van Knippenberg, (2004) une expérimentatrice imitait (ou n'imitait pas) le comportement des participants lors d'une interaction à la suite de quoi elle faisait «accidentellement» tomber des pièces de monnaie par terre. Les participants ayant été imités ont davantage aidé l'expérimentatrice à ramasser les pièces que les participants n'ayant pas été auparavant imités. L'imitation a donc pour conséquence d'entraîner une augmentation des comportements d'aide à l'égard de l'imité.

D'autres recherches ont montré que le fait d'imiter entraîne également davantage de comportements prosociaux à l'égard d'un autre individu que l'imitant. Dans leur expérience, Stel, van Baaren et Vonk (2008) donnaient pour consigne aux participants d'imiter (ou de ne pas imiter) un individu présenté à l'aide d'une vidéo. On précisait au groupe en condition d'imitation d'imiter les expressions faciales de la cible notamment en se focalisant sur l'observation des mouvements des yeux, des sourcils et de la bouche. A l'issu de cette première étape, un compère de l'expérimentateur abordait les participants et leur demandait de donner de l'argent pour une œuvre de charité. Il s'avère que les participants donnent significativement plus d'argent lorsqu'ils ont eu au préalable la consigne de se montrer imitant. Plusieurs recherches ont confirmé que les sollicitations d'aide émanant de personnes qui viennent de vous imiter sont plus fréquemment acceptées confirmant la robustesse de l'effet 
d'imitation sur le comportement d'aide à l'égard de la personne imitée (Fischer-Lokou, Martin, Guéguen \& Lamy, 2011; Guéguen, Martin, \& Meineri, 2011, Kulesza, Dolinski, Huisman, \& Majewski, 2014). Enfin, il semblerait que les comportements prosociaux entraînent une augmentation de l'imitation. Lorsque dans un premier temps les participants sont exposés à des mots (tâche d'amorçage) en lien avec les comportements d'aide à l'égard d'autrui, ils se montrent par la suite davantage imitants. (Leighton, Bird, Orsini \& Heyes, 2010).

\section{b) Comportements du consommateur}

Jacob, Guéguen, Martin et Boulbry (2011) observent que les individus ayant été imités par un vendeur de produits technologiques achètent davantage le produit conseillé par le vendeur que lorsque celui-ci n'a pas été imitant. Ces résultats ont été confirmés dans le cadre de la vente de produits cosmétiques (Kulesza, Szypowska, Jarman, \& Dolinski, 2014). Tanner, Ferraro, Chartrand, Bettman et van Baaren (2008) observent que l'imitation affecte également les comportements et les préférences des consommateurs. Dans leur première recherche, ils mettent en avant que le simple fait d'observer une personne en train de consommer un type particulier de biscuit augmentera significativement la probabilité pour que l'observateur lui-même consomme ce biscuit et le trouve par la suite meilleur. Dans deux autres recherches, ils montrent que le fait d'être imité par un individu présentant un produit (en l'occurrence une boisson pour la première expérience et des pailles au fromage pour la seconde) augmentera la préférence des individus imités à l'égard de ce produit et les conduira davantage à le consommer et à vouloir l'acheter. Il semble donc que l'imitation puisse être un outil efficace pour augmenter la consommation ainsi que les préférences des individus à l'égard d'un produit.

Martin (2010) a également montré que l'imitation verbale pouvait modifier les comportements alimentaires des enfants dans le cadre de la prévention de l'obésité infantile. En effet, après avoir été imités les enfants ont davantage consommé un fruit pour leur goûter, comme demandé par l'expérimentateur, ont consommé moins de sucreries et de sodas que les enfants non imités. Il semble également que cet effet de l'imitation soit durable puisque un an après l'expérimentation, les enfants imités déclarent davantage continuer à consommer un fruit pour le goûter que les enfants non imités.

\section{Les fonctions de l'imitation}




\subsection{L'imitation comme outil de communication}

Selon Lakin, Jefferis, Cheng et Chartrand (2003), l'imitation est préalable à la communication d'où son importance pour les espèces, avoir une capacité de perception et de décodage du comportement d'autrui serait un gage de survie. Chez l'humain, l'interaction sociale est certainement le comportement qui a permis la survie et l'adaptation de notre espèce. Un individu coopérant et maintenant des relations sociales positives avait plus de possibilité de survivre. Dans cette perspective, les comportements nonverbaux et automatiques pourraient avoir grandement facilité les interactions sociales. L'imitation serait ainsi devenue à ce point automatique et indispensable qu'elle aurait, selon Lakin et al (2003) le statut de véritable «glue sociale ». L'imitation sert donc de glue sociale en facilitant l'attachement et les liens, ainsi que l'engagement entre les individus (Maddux, Mullen \& Galinsky, 2008).

D'un point de vue évolutionniste, le lien entre affiliation et imitation montre que l'imitation a une fonction adaptative. L'imitation est une stratégie d'intégration au groupe et d'acceptation par autrui, ce qui favorise la survie sociale de l'imitant (Lakin, Jefferis, Cheng \& Chartrand, 2003). En raison de son caractère inscrit par l'évolution, et de son importance pour notre survie, l'imitation exercerait aujourd'hui son influence non plus pour notre survie physique ni même pour acquérir des comportements sociaux puisque l'apprentissage social passe par le langage mais resterait l'expression du désir d'affiliation à l'égard d'autrui: désir d'affiliation auquel nous réagirions de manière automatique et non-consciente.

Cette théorie évolutionniste est étayée par les travaux de Cheng et Chartrand (2003) qui montrent que les individus se montrent plus imitants envers des personnes proches d'eux ou ayant un statut plus élevé. En effet, selon ces auteurs, les individus auront tendance à imiter davantage et donc à concentrer leurs efforts d'affiliation sur les personnes susceptibles de les aider.

Selon Karremans et Vewijmeren (2008) l'imitation a une fonction sociale. Les auteurs montrent que l'on imite davantage une personne du sexe opposé si on veut établir une relation avec elle alors que si on se trouve déjà dans une relation sentimentale, on a tendance au contraire à produire moins d'imitation. Pour ces auteurs, l'imitation ici serait un processus non conscient impliqué dans des stratégies d'entrée ou au contraire d'évitement de la relation. Farley (2014) observe également une corrélation négative entre le niveau d'imitation d'hommes à l'égard d'une femme attrayante physiquement et le degré d'attachement 
sentimental au moment de l'expérimentation. Ceux-qui étaient libres ou peu engagés sentimentalement imitaient beaucoup tandis que ceux qui s'estimaient très engagées et amoureux imitaient peu. Pour cette chercheuse, l'imitation fonctionnerait comme un signal de distance sociale. Pour mettre de la distance on imiterait peu et pour se rapprocher nous imiterions davantage la personne.

\subsection{Imitation et empathie}

Pour Bavelas, Black, Lemery et Mullett (1986) l'imitation sert à signifier à ceux avec lesquels on interagit qu'on les comprend et qu'on sait ce qu'ils ressentent. Dans leur recherche, les auteurs ont remarqué que les individus imitent pour montrer aux autres qu'ils savent ce qu'ils ressentent et comment ils se sentent.

L'imitation sert à signifier aux individus avec lesquels on interagit qu'on comprend leurs émotions. Selon Barsalou, Niedenthal, Barbey et Ruppert (2003), l'imitation permet de reconnaître très rapidement les émotions ressenties par les autres notamment en imitant leurs expressions émotionnelles ce qui en retour entraîne une meilleure reconnaissance de l'émotion. Ainsi l'imitation servirait une fois de plus à créer ou à resserrer des liens entre les individus en leur permettant de mieux comprendre les émotions ressenties par ces derniers.

\subsection{Imitation et similarité}

Dans une expérience, Ramanathan et McGill (2007) ont remarqué que l'imitation des expressions faciales et la contagion émotionnelle qu'elle engendre permettent aux individus d'évaluer une expérience de la même façon que celui qui l'a réalisée. Il semblerait que l'imitation conduise les individus imitants à partager davantage les opinions des individus imités que l'absence d'imitation. L'imitation engendre donc une plus grande similarité des attitudes.

D'un autre côté, van Swol et Drury (soumis) ont montré que le lien entre imitation et convergence d'attitudes n'était pas unidirectionnel. En effet, l'imitation entraîne une plus forte convergence d'opinions et à l'inverse, une plus forte convergence d'opinions engendre une augmentation de l'imitation. Scheflen (1964) et LaFrance (1982) avaient déjà évoqué dans leurs travaux respectifs le fait que l'imitation puisse être la conséquence d'un point de vue partagé. 


\section{Conclusion}

Chartrand et Bargh (1999) qualifient l'imitation d'effet caméléon, puisque selon eux nous imitons ce que nous percevons dans notre environnement pour nous mettre en harmonie avec celui-ci et ce de manière non consciente et automatique. Nous changeons nos comportements pour les mettre en concordance avec l'environnement social dominant. L'imitation non consciente des autres fait partie intégrante de notre fonctionnement et de notre existence au quotidien. C'est un aspect fondamental de nos interactions avec les autres. L'imitation sert à créer et à renforcer des liens entre les individus et sert également à réguler leurs interactions sociales. Nous n'avons pas conscience du rôle important joué par l'imitation et pourtant il semble que nous sommes capables de l'utiliser à des fins très personnelles. De manière implicite, il semble que les individus aient compris que l'imitation pouvait les aider à créer ou à renforcer des liens avec les autres. L'imitation a une fonction adaptative, elle sert véritablement de "glue sociale» en liant et soudant les individus entre eux (Lakin et al., 2003).

Nous avons vu également qu'il existe des facteurs motivationnels, contextuels et individuels qui facilitent ou inhibent les effets de l'imitation. Il est fortement probable que d'autres facteurs puissent entrer en jeu dans le mécanisme de l'imitation et cela reste encore à découvrir. D'après les travaux de Bandura (1986), l'imitation lorsqu'elle est utilisée volontairement par un individu, lorsqu'elle est consciente, est essentielle à l'apprentissage et la compréhension de notre système social. L'imitation pourrait aussi être mise en place stratégiquement, de façon intentionnelle (Maddux, Mullen, \& Galinsky, 2008).

Une synthèse des principaux effets de l'imitation est présentée dans les Tableaux 1, 2 et 3 ci-après. Chaque tableau différencie la situation selon laquelle l'individu est imité, l'individu est conduit à imiter ou l'individu est conduit à inhiber son comportement d'imitation.

$$
\begin{aligned}
& \text {-Tableau } 1 \text { ici - } \\
& \text {-Tableau } 2 \text { ici - } \\
& \text {-Tableau } 3 \text { ici - }
\end{aligned}
$$

La recherche sur l'imitation en est à ses débuts et il reste encore beaucoup à découvrir notamment concernant les mécanismes qui la sous-tendent, ainsi que les facteurs susceptibles d'inhiber ses effets, voire même de les annuler, ou au contraire les facteurs facilitant et augmentant le niveau d'imitation. De même il reste beaucoup à apprendre des conséquences 
de l'imitation sur les comportements. Nous pouvons notamment nous interroger sur l'impact du statut social sur l'imitation. En effet nous pouvons penser qu'un individu aura tendance à moins imiter un autre individu lorsque ce dernier à un statut social inférieur. Il serait également intéressant de mesurer l'impact de l'imitation de la part d'un individu ayant un statut social non valorisé, ayant un stigma ou un handicap par exemple. Il serait intéressant de mesurer quels effets entraînent, sur le jugement social et les comportements, l'imitation d'un individu auquel on ne souhaiterait pas s'affilier ou ressembler.

Les prochaines recherches pourront également s'attacher à essayer d'identifier les limites de l'imitation. En effet, il serait intéressant d'observer si l'induction d'une imitation forte (imitation systématique des comportements ou des phrases des participants) entraîne des effets plus forts sur le comportement et les attitudes que l'induction d'une imitation faible. Nous pouvons nous demander s'il y a un niveau plafond de l'imitation au-delà duquel nous n'observerions plus d'effet, voire même des effets contraires (tels par exemple que la réactance psychologique, Brehm 1966).

Tanner et al. (2008) observent, dans leur expérience, une plus grande soumission et un changement d'attitude à l'égard de produits présentés par l'expérimentateur imitant. Les auteurs expliquent ces effets par l'empathie que l'imitation génèrerait chez les individus imités à l'égard des individus imitant. Toutefois, aucune recherche n'a jusqu'à présent véritablement mesuré l'empathie ressentie par les sujets à l'issue de l'interaction durant laquelle ils ont été ou non imités. Il serait pertinent de mesurer, dans de prochaines études, si l'imitation engendre effectivement de l'empathie (test du C.E.C27. de Favre, Joly, Reynaud et Laurent, 2005). S'il s'avère qu'il y a un lien entre l'imitation et l'empathie, il s'agira d'observer si ce lien est direct ou s'il est médiatisé.

Les prochaines expérimentations portant sur l'imitation devraient également tenter de manipuler le besoin d'aide manifesté par les expérimentateurs, à l'instar de l'expérience de Tanner et al. (2008) sur les préférences et la consommation alimentaires. En effet, dans leur expérience, les auteurs manipulaient l'imitation et observaient que plus l'expérimentateur imitant manifeste son besoin d'aide, plus les individus imités lui rendent service et abondent dans son sens (en évaluant son produit plus favorablement, en le consommant plus et en désirant davantage l'acheter). Les auteurs expliquent leurs résultats par l'empathie générée par l'imitation. Il serait donc intéressant de voir si les sujets imités modifieraient davantage encore leurs comportements (à court et à long termes) dans le sens attendu par l'expérimentateur si ce dernier leur déclarait explicitement avoir besoin d'eux. La similarité entraîne davantage 
d'empathie, il serait donc intéressant d'observer si l'induction d'une similarité entre deux individus couplée à la mise en évidence d'un besoin manifeste entraînerait davantage d'imitation.

Les effets de l'imitation sur les comportements d'aide, de proximité et les préférences à l'égard des individus pourraient être expliqués par le sentiment de familiarité qui naîtrait chez la personne imitée à l'égard de la personne imitant (Guéguen et al., 2009). Il s'agira donc, dans de prochaines recherches, de mesurer le sentiment de familiarité ressenti à l'égard des individus imitants. De surcroît, nous avons montré qu'une personne se montre plus imitante à l'égard d'une autre personne avec laquelle elle croit partager des caractéristiques communes, telles que, par exemple, le même nom de famille. Ainsi, la similarité perçue entre les individus entraînerait un sentiment de familiarité réciproque plus important et donc des liens d'autant plus forts ce qui augmenterait le niveau d'imitation entre ces derniers. Il serait donc intéressant d'évaluer le sentiment de familiarité ressenti à l'égard de l'individu les ayant préalablement imités de manière à voir si la familiarité médiatise les effets de la similarité sur l'imitation.

Pour finir, il serait également intéressant de rapprocher les travaux portant sur l'imitation du modèle de L'ELM (Petty \& Cacioppo 1979) pour observer quel traitement de l'information est mis en place par les sujets imités. Nous pouvons en effet nous demander si l'imitation comme les travaux sur l'engagement entraîne un traitement systématique des messages puisque les sujets sont plus motivés à les traiter. Au contraire, dans la mesure où l'imitation renvoie à l'individu imité qu'on ne le juge pas, qu'on ressent de l'empathie pour lui, qu'on souhaite créer des liens avec lui, pourrait entraîner un traitement heuristique des messages.

\section{Références}

Achaibou, A., Pourtois, G., Schwartz, S. \& Vuilleumier, P. (2008). Simultaneous recording of EEG and facial muscle reactions during spontaneous emotional mimicry. Neuropsychologia, 46(4), 1104-1113.

Ashton-James, C. E., \& Chartrand, T.L. (2009). Social cues for creativity: The impact of behavioral mimicry on convergent and divergent thinking, Journal of Experimental Social Psychology, 45(4), 1036-1040.

Ashton-James, C. E., \& Levordashka, A. (2013). When the wolf wears sheep's clothing: Individual differences in the desire to be liked influence nonconscious behavioral mimicry. Social Psychological and Personality Science, 4 643-648.

Ashton-James, C.E., van Baaren, R.B., Chartrand, T.L. \& Decety, J., Karremans, J.C. (2007). 
Mimicry and me: The impact of mimicry on self-construal. Social Cognition.

Bailenson, J. N. \& Yee, N. (2005). Digital chameleons: Automatic assimilation of nonverbal gestures in immersive virtual environments. Psychological Science, 16, 814-819.

Bandura, A., (1986). Social Foundations of Thought and Action. Prentice-Hall, Englewood Cliffs, NJ.

Bargh, J. A., Chen, M. \& Burrows, L. (1996). Automaticity of social behavior: Direct effects of trait construct and stereotype priming on action. Journal of Personality and Social Psychology, 71, 230-244.

Barsalou, L.W., Niedenthal, P.M., Barbey, A., \& Ruppert, J. (2003). Social Embodiment. In B. Ross (Ed.), The Psychology of Learning and Motivation, 43, 43-92. San Diego: Academic Press.

Baumeister, R. F. \& Leary, M. R. (1995). The need to belong: Desire for interpersonal attachments as a fundamental human motivation. Psychological Bulletin, 117, 497529.

Bavelas, J. B., Black, A., Chovil, N., Lemery, C. R. \& Mullett, J. (1988). Form and function in motor mimicry. Topographic evidence that the primary function is communicative. Human Communication Research, 14, 275-299.

Bavelas, J.B., Black, A., Lemery, C. R., MacInnis, S. \& Mullett, J. (1986). Experimental methods for studying "elementary motor mimicry." Journal of Nonverbal Behavior, $10,102-119$.

Bavelas, J.B., Black, A., Lemery, C. R. \& Mullett, J. (1986). "I show how you feel." Motor mimicry as a communicative act. Journal of Personality and Social Psychology, 50, 322-329.

Bavelas, J.B., Black, A., Lemery, C.R. \& Mullett, J. (1987). Motor mimicry as primitive empathy. In N. Eisenberg \& J. Strayer (Eds.), Empathy and its development (pp. 317338). Cambridge, UK: Cambridge University Press.

Bergmann, H., Jansen, C., Muller, B. \& Wagenaar, A. (2005). Does imitation help to improve your impression when being disliked? Helpt imitatie je indruk te verbeteren wanneer je onaardig gevonden wordt? In E. Gordijn, J. Ouwerkerk , R. Holland \& A. Meijnders (Ed.), Jaarboek Sociale Psychologie 2004. Delft: ASPO Pers.

Bekkering, H., Wohlschläger, A. \& Gattis, M. (2000). Imitation of gestures in children is goal-directed. Quarterly Journal of Experimental Psychology, 53, 153-164.

Berkowitz, L. (1984). Some effects of thoughts on the anti- and prosocial influences of media events: A cognitive neoassociationistic analysis. Psychological Bulletin, 95, 410-427.

Bernieri, F.J. (1988). Coordinated movement and rapport in teacher-student interactions. Journal of Nonverbal Behavior, 12, 120-138. 
Bernieri, F.J. \& Rosenthal, R. (1991). Interpersonal coordination : behavior matching and interactional synchrony. In : Feldman, R. \& Rimé, B. (eds). Fundamentals of nonverbal behavior: Studies in emotion \& social interaction. New York : Cambridge University Press.

Blairy, S., Herrera, P. \& Hess, U. (1999). Mimicry and the judgment of emotional facial expressions. Journal of Nonverbal behavior, 23, 5-41.

Bock, J. K. (1989). Closed-class immanence in sentence production. Cognition 31, 163-186.

Brass, M., Bekkering, H., \& Prinz, W. (2001). Movement observation affects movement execution in a simple response task. Acta Psychologica, 106, 3 - 22.

Brewer, M. B., (1991). The social self: On being the same and different at the same time. Personality and Social Psychology Bulletin, 17, 475-482.

Bush, L.K., Barr, C.L., McHugo G.J. \& Lanzetta, J.T. (1989). The effects of facial control and facial mimicry on subjective reaction to comedy routines. Motivation and emotion, 12, 120-138.

Carpenter, M., Akhtar, N., \& Tomasello, M. (1998). Fourteen- to 18-month-old infants differentially imitate intentional and accidental actions. Infant Behavior and Development, 21, 315-330.

Carr, L., Iacoboni, M., Dubeau, M.-C., Mazziotta, J. C. \& Lenzi, G. L. (2003). Neural mechanisms of empathy in humans: a relay from neural systems for imitation to limbic areas, Proceedings of the National Academy of Sciences, 100(9), 5497-5502.

Castelli, L., Pavan, G., Ferrari, E. \& Kashima, Y. (2009). The stereotyper and the chameleon: The effects of stereotype use on perceivers' mimicry. Journal of Experimental Social Psychology, 45, 835-839.

Chartrand, T. L. \& Bargh, J. A. (1999). The chameleon effect: The perception-behavior link and social interaction. Journal of Personality and Social Psychology, 76, 893-910.

Chartrand, T.L., Cheng, C.M., \& Jefferis, V.E. (2002). You're just a chameleon: The automatic nature and social significance of mimicry. In M. Jarymowicz \& R.K. Ohme (Eds.) Natura automatyzmow (Nature of Automaticity; pp. 19-23). Warszawa: IPPAN \& SWPS.

Chartrand, T.L. \& Dalton, A.N. (2009). Mimicry: Its ubiquity, importance, and functionality.In J. Bargh, P. Gollwitzer \& E. Morsella (Eds.), Psychology of Action, Vol. 2. (pp 458 - 483). New York: Guilford.

Chartrand, T. L \& Jefferis, V. (2003). Consequences of automatic goal pursuit and the case of nonconscious mimicry. In J. P. Forgas, K. D. Williams \& W. Von Hippel (Eds.). Responding to the social world: Implicit and explicit processes in social judgments and decisions. Philadelphia: Psychology press. 
Chartrand, T. L., Kawada, C. L. K. \& Bargh, J. A. (2002). It takes one to know one: When your own behavior influences your judgment of others. Unpublished manuscript

Chartrand, T.L., Maddux, W. \& Lakin, J. (2005). Beyond the perception-behavior link: The ubiquitous utility and motivational moderators of nonconscious mimicry. In R. Hassin, J. Uleman, \& J.A. Bargh (Eds.), The New Unconscious (pp. 334-361). New York: Oxford University Press.

Chartrand, T.L. \& van Baaren, R. (2009). Human mimicry. To appear in To appear in M. Zanna (Ed.), Advances in Experimental Social Psychology, 41, 219-274.

Cheng, C. M. \& Chartrand, T. L. (2003). Self-monitoring without awareness: Using mimicry as a nonconscious affiliation strategy. Journal of Personality and Social Psychology.

Chen, M., Chartrand, T.L., Lee-Chai, A.Y. \& Bargh, J.A. (1998). Priming primates: Human and otherwise. Behavioral and Brain Sciences, 21(5), 685-686.

Cialdini, R. B. (2004). Influence et manipulation : comprendre et maîtriser les mécanismes et les techniques de persuasion. First éditions : Paris.

Cross, S. E. \& Madson, L. (1997). Models of the self: Self-construals and gendre. Psychological Bulletin, 122, 5-37.

Dabbs, J. M. (1969). Similarity of gestures and interpersonal influence. Proceedings of the 77th Annual Convention of the American Psychological Association, 4, 337-338.

Dalton, A.N., Chartrand, T. L. \& Finkel, E. L. (2010). The Shema-Driven Chameleon: How mimicry affects executive and self regulatory resources. Journal of Personality and Social Psychology.

Davis, M. H. (1983). Measuring individual differences in empathy: Evidence for a multidimensional approach. Journal of Personality and Social Psychology, 44, 113126.

Decety, J., Jeannerod, M., Germain, M. \& Pastene, J. (1991). Vegetative response during imagined movement is proportional to mental effort. Behavioural Brain Research, 42, $1-5$.

Dijksterhuis, A. \& Bargh, J.A. (2001). The perception-behavior Expressway: Automatic effects of social perception on social behavior. Advances in Experimental Social Psychology, 33, 1-40.

Dimberg, U., Andréasson, P., \& Thunberg, M. (2011). Emotional empathy and facial reactions to facial expressions. Journal Of Psychophysiology, 25(1), 26-31.

Dimberg, U. \& Thunberg, M. (1998). Rapid facial reactions to different emotionally relevant stimuli. Scandinavian Journal of Psychology, 39, 39-45.

Dimberg, U., Thunberg, M. \& Elmehed, K. (2000). Unconscious facial reactions to emotional facial expressions. Psychological Science, 11, 86-89. 
Estow, S., Jamieson, J. P. \& Yates, J. R. (2007). Self-monitoring and mimicry of positive and negative social behaviors. Journal of Research in Personality, 41, 425-433.

Farley, S. D. (2014). Nonverbal reactions to an attractive stranger: The role of mimicry in communicating preferred social distance. Journal of Nonverbal Behavior, 38, 195208.

Fischer-lokou J., Guéguen, N., Lamy, L., Martin, A., Bulllock, A. (2014) Imitation in mediation: Effects of the duration of mimicry on reaching agreement, Social Behavior and Personality, 42, 189-196.

Fischer-Lokou, J., Martin A., Guéguen, N., \& Lamy, L. (2011). Mimicry and propagation of prosocial behavior in a natural setting. Psychological Reports.108, 599-605.

Fadiga L., Fogassi L., Pavesi G. \& Rizzolatti G. (1995). Motor facilitation during action observation: a magnetic stimulation study. Journal of Neurophysiology, 73(6), 26082611.

Friedman, H. S. \& Riggio, R. (1981). The effect of individual differences in nonverbal expressiveness on transmission of emotion. Journal of Nonverbal Behavior, 6, 96104.

Gallese, V., Fadiga, L., Fogassi, L. \& Rizzolatti, G. (1996). Action recognition in the premotor cortex. Brain, 119, 593-609.

Gattis, M. (2002). Imitation is mediated by many goals, not just one. Developmental Science, $5,27-29$.

Giles, H. \& Powesland, P. F. (1975). Speech style and social evaluation. London: Academic Press.

Guéguen N. (2009). Mimicry and Women Attractiveness: An Evaluation in a Courtship Context. Social Influence, 4(4), 249-255.

Guéguen N. (2009). The mimicker is the mirror of myself: Impact of mimicry on selfconsciouness and social anxiety. Social Behavior and Personality, 39, 725-728.

Guéguen N., (2013). Mimicry and honesty: People give more honest responses to their mimicker. International Journal of Psychological Research, 6, 53-58.

Guéguen, N., Jacob, C. \& Martin, A. (2009). Mimicry in social interaction: Its effect on human judgment and behavior. European Journal of Social Sciences, 8(2), 253-259.

Guéguen, N. \& Martin, A. (2008). L'effet de 1' imitation sur l'évaluation d'autrui : une expérimentation dans un contexte de séduction. Revue Internationale de psychologie sociale, 21(4), 5-24.

Guéguen, N. \& Martin, A. (2009). Incidental similarity facilitates behavioral mimicry. Social Psychology. Vol 40(2), 88-92. 
Guéguen, N., Martin, A., \& Meineri, S. (2011). Mimicry and altruism: An evaluation of mimicry on explicit helping requests. The Journal of Social Psychology, 151, 1-4.

Guéguen N., Martin A., Meineri S., \& Simon J., (2013). Using mimicry to elicit answers to intimate questions in survey research. Field Methods, 25, 47-57.

Guéguen N. Martin A. \& Vion M. (2009). L'effet d'une similarité fortuite entre deux individus sur le comportement d'imitation. Psychologie Française, 54, 337-353.

Guilford, J.P. (1959). Creativity. American Psychologist, 5, 444-454.

Harakeh, Z., Engels, R., van Baaren, R. B. \& Scholte, R. H. J. (2007). Imitation of cigarette smoking: An experimental study of smoking in a naturalistic setting. Drug and Alcohol Dependence, 86, 199-206.

Hatfield, E., Cacioppo, J T., \& Rapson, R. L. (1992). Primitive emotional contagion. In M. S. Clark (Ed.), Emotion and Social Behavior, 14, 151-177.

Hatfield, E., Rapson, R.L. \& Le, Y.L. (2011). Emotional contagion and empathy. In J. Decety and W. Ickes (Eds.) The social neuroscience of empathy. Boston, MA: MIT Press.

Haviland, J. M. \& Lelwica, M. (1987). The induced affect response: 10-week-old infants' responses to three emotion expressions. Developmental Psychology, 23(1), 97-104.

Hess, U. \& Blairy, S. (2001). Facial mimicry and emotional contagion to dynamic emotional facial expressions and their influence on decoding accuracy. International Journal of Psychophysiology, 40, 129-141.

Hsee, C. K., Hatfield, E., Carlson, J. \& Chemtob, C. (1990). The effect of power on susceptibility to emotional contagion. Cognition and Emotion, 4, 327-340.

Iacoboni, M., Woods, R.P., Brass, M., Bekkering, H., Mazziotta, J.C. \& Rizzolatti, G. (1999). Cortical mechanisms of human imitation. Science, 286, 2526-2528.

Inzlicht, M., Gutsell, J. N., Legault, L. (2012). Mimicry reduces racial prejudice. Journal of Experimental Social Psychology, 48, 361-365.

Jacob, C., \& Guéguen N. (2013) The Effect of employees' verbal mimicry on tipping. International Journal of Hospitality Management, 35, 109-111.

Jacob, C., Guéguen, N., Martin, A., \& Boulbry, G. (2011). Retail salespeople's mimicry of customers: Effects on consumer behavior. Journal of Retailing and Consumer Services, 18, 381-388.

Jefferis, V. E., van Baaren, R. \& Chartrand, T. L. (2009). The functional purpose of mimicry for creating interpersonal closeness. Manuscript in preparation, The Ohio State University.

Johnston, L., (2002). Behavioral Mimicry and Stigmatization. Social Cognition, 20, 18-35. 
Kaitz, M., Meschulach-Sarfaty, O., Auerbach, J. \& Eidelman., A. (1988). A reexamination of newborn's ability to imitate facial expressions. Developmental Psychology, 24, 3-7.

Karremans, J. C. \& Verwijmeren, T. (2008). Mimicking attractive opposite-sex others: The role of romantic relationship status. Personality and Social Psychology Bulletin, 34, 939-950.

Keysers, C., \& Gazzola, V. (2010). Social neuroscience: Mirror neurons recorded in humans. Current Biology, 20, 353-354.

Korb, S., Malsert, J., Rochas, V., Rihs, T. A., Rieger, S. W., Schwab, S., Niedenthal, P. M., Grandjean, D. (2015). Gender differences in the neural network of facial mimicry of smiles-An rTMS study. Cortex, 70, 101-114.

Kouzakova, M. (2009). Subtle signs of exclusion: how lack of mimicry affects the self and other perception. Social Psychology Thesis. Netherlands : Radboud University Nijmegen, 2009, 128 p.

Kouzakova, M., Karremans, J. C., Van Baaren, R. B., \& Van Knippenberg, A. (2010). A stranger's cold shoulder makes the heart grow fonder: Why not being mimicked by a stranger enhances longstanding relationship evaluations. Social Psychological and Personality Science. 1, 87-93.

Kulesza, W., Cislak, A., Vallacher, R. R., Nowak, A., Czekiel, M., \& Bedynska, S. (2015). The face of the chameleon: The experience of facial mimicry for the mimicker and the mimickee. Journal of Social Psychology, 1-15.

Kulesza, W., Dolinski, D., Huisman, A., \& Majewski, R. (2014). The echo effect: The power of verbal mimicry to influence pro-social behavior. Journal of Language and Social Psychology, 33, 185-203.

Kulesza, W., Szypowska, Z., Jarman, M., \& Dolinski, D. (2014). Attractive chameleons sell: The mimicry-attractiveness link. Psychology \& Marketing, 31, 549-561.

LaFrance, M. (1979). Nonverbal synchrony and rapport: Analysis by the cross-lag panel technique Social Psychology Quaterly, 42, 66-70.

LaFrance, M. (1982). Posture mirroring and rapport. In M. Davis (Ed.), Interaction rhythms: Periodicity in commutative behavior (pp. 279-298). New York: Human Sciences Press.

LaFrance, M. \& Broadbent, M. (1976). Group rapport: Posture sharing as a nonverbal indicator. Group and Organization Studies, 1, 328-333.

Laird, J. D., Alibozak, T., Davainis, D., Deignan, K., Fontanella, K., Hong, J., Brett, L. \& Pacheco, C. (1994). Individual differences in the effects of spontaneous mimicry on emotional contagion. Motivation and Emotion, 18, 231-245.

Lakin, J., L. \& Chartrand, T. L. (2003). Using nonconscious behavioral mimicry to create affiliation and rapport. Psychological Science, 14, 334-339. 
Lakin, J.L. \& Chartrand, T.L. (2005). Exclusion and nonconscious behavioral mimicry. In J.P. Forgas, K. D. Williams \& W. von Hippel (Eds.) The social outcast: Ostracism, social exclusion, rejection, and bullying. New York: Psychology Press.

Lakin, J. L., Chartrand, T. L. \& Arkin, R. M. (2008). I am too just like you: Nonconscious mimicry as an automatic behavioral response to social exclusion. Psychological Science, 19, 816-822.

Lakin, J. L., Jefferis, V. E., Cheng, C. M. \& Chartrand, T. (2003). The chameleon effect as social glue: Evidence for the evolutionary significance of nonconscious mimicry Journal of Nonverbal Behavior, 27, 145-162.

Leander, N. P., Chartrand, T. L. \& Wood, W. (2011). Mind your mannerisms: Behavioral mimicry elicits stereotype conformity. Journal of Experimental Social Psychology, 47, 195-201.

Leighton, J., Bird, G., Orsini, C. \& Heyes, C.M. (2010) Social attitudes modulate automatic imitation. Journal of Experimental Social Psychology 46, 905-910.

Levelt, W.J.M. \& Kelter, S. (1982) Surface form and memory in question answering. Cognitive Psychology 14, 78-106.

Lipps, T. (1903). Einfühlung, innere Nachahmung und Organempfindungen, Archiv für die gesamte Psychologie, vol 1, trad. angl. Empathy, inner imitation, and sense-feelings, in Melvin Rader (éd.), A Modern Book of Esthetics. An Anthology, 5e éd., Holt - Rinehart \& Winston, 1979, pp. 371-378.

Lott, A.J. \& Lott, B.E. (1961). Group cohesiveness, communication level, and conformity. Journal of Abnormal and Social Psychology, 62, 408-412.

Love, J. L. \& Parker-Robinson, C. (1972). Children's imitation of grammatical and ungrammatical sentences. Child Development, 43(2), 309-319.

Lundquist, L.O. \& Dimberg, U. (1995). Facial expressions are contagious. Journal of Psychophysiology, 9, 203-211.

Maddux, W.W., Mullen, E. \& Galinsky, A.D. (2008). Chameleons bake bigger pies and take bigger pieces: Strategic behavioral mimicry facilitates negotiation outcomes. Journal of Experimental Social Psychology, 44(2), 461-468.

Martin (2010). L'effet Caméléon comme outil de prévention de l'obésité infantile. L'imitation, une nouvelle technique de soumission comportementale? (thèse de doctorat inédite). Université de Bretagne Sud.

Martin, A., Fischer-Lokou, J. \& Guéguen, N. (in press). Impact of verbal mimicry on children fruit consumption. Food Quality and Preferences.

Martin, A. \& Guéguen, N. (2015). Repeating what children say positively influences their learning and motivation. Learning \& Motivation, 52, 48-53. 
Martin A., Guéguen, N. \& Fischer-Lokou J. (2010). The impact of guilt on mimicry behavior. Social Behavior and Personality, 38(7), 987-992.

Mathersul, D., McDonald, S., \& Rushby, J. A. (2013). Understanding advanced theory of mind and empathy in high-functioning adults with autism spectrum disorder. Journal Of Clinical And Experimental Neuropsychology, 35(6), 655-668.

Maurer, R. E. \& Tindall, J. H. (1983). Effects of postural congruence on client's perception of counselor empathy. Journal of Counseling Psychology, 30, 158-163.

Meltzoff, A. N (1988). Infant imitation after a 1-week delay. Long-term memory for novel acts and multiple stimuli. Developemental Psychology, 24, 470-467.

Meltzoff, A. N. (1995). Understanding the intentions of others: Re-enactment of intended acts by 18-month-old children. Developmental Psychology, 31, 838-850.

Meltzoff A. et Decety J. (2003), What imitation tells us about social cognition: a rapprochement between developmental psychology and cognitive neuroscience, Philosophical Transactions of the Royal Society: Biological Sciences, 358, 491-500.

Meltzoff, A. N. \& Moore, M. K. (1977). Imitation of facial and manual gestures by human neonates. Science, 198, 75-78.

Meltzoff, A. N. \& Moore, M. K. (1995). Imitation memory, and the representation of persons. Infant Behavior and Development, 17, 83-99.

Moore, M. M. (1995). Courtship signaling and adolescents: "Girls just wanna have fun"? The journal of Sex Research, 32, 319-328.

Neuberg, S.L., Smith, D.M., Hoffman, J.C. \& Russell, F.J. (1994). When we observe stigmatizedand "normal" individuals interacting: Stigma by association. Personality and Social Psychology Bulletin, 20, 196-209.

Neumann, R. \& Strack, F. (2000). "Mood contagion": The automatic transfer of mood between persons. Journal of Personality and Social Psychology, 79, 211-223.

Over, H. \& Gattis, M. (2011). Verbal imitation is based on intention understanding. Coginitive Development, 25, 46-55

Parks, G., (1980). Social Determinants of Alcohol Consumption: Effects of Social Atmosphere on Modeling of Alcohol Consumption. Unpublished Doctoral Dissertation, University of Washington.

Platek, S. M., Critton, S. R., Myers, T. E. \& Gallup, G. G. (2003). Contagious yawning: The role of self-awareness and mental state attribution. Cognitive, Brain Research, 17, 223-227.

Provine, R. R. (1992). Faces as releasers of contagious yawning: An approach to face detection using normal human subjects. Bulletin of the Psychonomic Society, 27, 211214. 
Quigley, B. M. \& Collins, R. L. (1999). Modeling of alcohol consumption: A meta-analytic review. Journal of Studies on Alcohol, 60, 90-98.

Ramanathan, S. \& McGill, A. L. (2007). Consuming with Others: Social Influences on moment-to-Moment and Retrospective Evaluations of an Experience. Journal of Consumer Research, 34(4), 506-524.

Redeker, M., Stel, M. \& Mastop, J. (2011). Does mimicking others change your self-view? Journal of Social Psychology, 151, 387-390.

Rizzolatti G., Fogassi L. \& Gallese V. (2001). Neurophysiological mechanisms underlying the understanding and imitation of action. Nature Reviews Neuroscience, 2:661-667.

Rizzolatti G. et Sinigaglia C. (2007), Les neurones miroirs, Paris, Odile Jacob.

Rychlowska, M., Canadas, E., Wood, A., Krumhuber, E. G., Fischer, A., \& Niedenthal, P. M. (2014). Blocking mimicry makes true and false smiles look the same. PLOS ONE, 26;9(3)e90876.

Scheflen, A. E. (1964). The significance of posture in communication systems. Psychiatry, $27,316-331$.

Sebanz, N., Knoblich, G. \& Prinz, W. (2003). Representing others' actions: Just like one's own? Cognition, 88, B11-B21.

Simner, M. L. (1971). Newborn's response to the cry of another infant. Developmental Psychology, 5, 136-150.

Sonnby-Borgström, M. (2002). Automatic mimicry reactions as related to differences in emotional empathy. Scandinavian Journal of Psychology, 43, 433-443.

Stel, M., Bergmann, H., Jansen, C., Muller, B. \& Wagenaar, A. (2005). Does imitation help to improve your impression when being disliked? Helpt imitatie je indruk te verbeteren wanneer je onaardig gevonden ordt? In E. Gordijn, J. Ouwerkerk, R. Holland \& A. Meijnders (Ed.), Jaarboek Sociale Psychologie 2004.

Stel, M. Blascovich, J. mc Call, C., Mastop, J., van Baaren, R.B. \& Vonk, R. (2009). Mimicking disliked others: Effects of a priori liking on the mimicry-liking link. European Journal of Social Psychology, 40(5), 867-880.

Stel, M., van Baaren, R. B., \& Vonk, R. (2008). Effects of mimicking: acting prosocially by being emotionally moved. European Journal of Social Psychology, 38, 965-976.

Stel, M. \& van Knippenberg, A. (2008). The role of facial mimicry in the recognition of affect. Psychological Science, 19(10), 984-985.

Stel, M., van Dijk, E. \& Olivier, E. (2009). You want to know the truth? Then don't mimic! Psychological Science.

Stel, M., Vonk, R., van Baaren, R. B. \& Smeets, R. C. (2009). The social consequences of 
mimicry: Effects on empathy and bonding. Manuscript submitted for publication.

Stel, M. \& Vonk, R. (2010). Mimicry in social interaction: Benefits for mimickers, mimickees, and their interaction. British Journal of Psychology, 101, 311-323..

Sullins, E.S. (1991). Emotional contagion revisited: Effects of social comparison and expressive style on mood convergence. Personality and Social Psychology Bulletin, $17,166-174$

Tanner, R. J., Ferraro, R., Chartrand, T. L., Bettman, J. R. \& van Baaren, R.B. (2008). Of Chamelon and consumption: The impact of mimicry on choice and preferences. Journal of Consumer Research, 34(6), 754-766.

Tarde, G. (2001). Les lois de l'imitation. Paris : Les empêcheurs de penser en rond. (éd. originale 1890).

Termine, N. T. \& Izard, C. E. (1988). Infants' response to their mother's expressions of joy and sadness. Developmental Psychology, 24, 223-229.

Uldall, B., Hall, C., \& Chartrand, T. (2008). Optimal distinctiveness and mimicry. Manuscrit en préparation, University of Hawaii.

Van Baaren, R. B., Holland, R. W. \& van Knippenberg, A. (2002). Mimicry and interpersonal closeness. Manuscrit non publié.

Van Baaren, R. B., Maddux, W. W., Chartrand, T. L., de Bouter, C. \& van Knippenberg, A. (2003). It takes two mimic: Behavioral consequences of self-construals. Journal of Personality and Social Psychology, 84, 1093-1102.

Van Baaren, R. B., Holland, R. W., Steenaert, B. \& van Knippenberg, A. (2003). Mimicry for money: Behavioral consequences of imitation. Journal of Experimental Social Psychology, 39, 393-398.

Van Baaren, R. B., Holland, R. W., Kawakami, K. \& van Knippenberg, A. (2004). Mimicry and prosocial behaviour. Psychological Science, 14, 71-74.

Van Baaren, R. B., Horgan, T. G., Chartrand, T. L. \& Dijkmans, M. (2004). The forest, the trees, and the chameleon: Context dependence and mimicry. Journal of Personality and Social Psychology, 86(3), 453-459.

Van Baaren, R. B., Fockenberg, D., Holland, R. W., Jansen, L. \& van Knippenberg, A. (2006). The moody chameleon: The effect of mood on non-conscious mimicry. Social Cognition, 24, 426-437.

Van Straaten, I. Engels, R., Finkenauer, C. \& Holland, R.W (2008). Sex differences in shortterm mate preferences and mimicry: A Semi-Naturalistic Experiment. Archives of Sexual Behavior, 37, 902-911.

Van Swol, L. (2003) The effects of nonverbal mirroring on perceived persuasiveness, agreement with an Imitator, and reciprocity in a group discussion. Communication 
Research, 30(4), 461-480.

Van Swol, L. M. \& Drury, M. (Manuscrit en progression). Effects of nonverbal imitation on perceptions of imitator's persuasiveness and knowledge. University of Wisconsin-Madison.

Van Swol, L. M. \& Drury. M. (soumis). The effects of shared opinions on nonverbal mimicry. Social Psychological and Personality Science.

Webb, J.T. (1969). Subject speech rates as a function of interviewer behavior. Language and speech, 12, 54-67.

Webb, J.T. (1972). Interview Synchrony: An Investigation of Two Speech Rate Measures. In A.W. Siegman and B. Pope (Eds.), Studies in Dyadic Communication pp.115-133). New York: Pergamon Press.

Yabar, Y., Johnston, L., Miles, L. \& Peace, V. (2006). Implicit behavioral mimicry: Investigating the impact of group membership. Journal of Nonverbal Behavior, 30, 97-113.

Young, R. D. \& Frye, M (1966). Some are laughing; some are not: Why? Psychological Reports, 18, 747-752.

Zajonc, R. B., Adelmann, K. A., Murphy, S. T. \& Niedenthal, P. M. (1987). Convergence in the physical appearance of spouces. Motivation and Emotion, 11, 335-346. 
Tableau 1: Tableau de synthèse des principaux effets de l'imitation lorsque l'individu est imité par autrui

\begin{tabular}{|c|c|}
\hline Registre d'influence & Effets constatés et références \\
\hline Jugement d'autrui & $\begin{array}{l}\text { Un conseiller qui a imité ses clients est jugé comme ayant plus d'empathie (Maurer \& } \\
\text { Tindall, 1983). } \\
\text { Une personne qui nous imite est jugée plus positivement (Chartrand et Bargh, 1999; } \\
\text { Kulesza et al., 2015) } \\
\text { On fait plus confiance à une personne qui nous imite (Van Swol, 2003) } \\
\text { Un vendeur qui nous a imité est jugé plus compétent, serviable à l'écoute et proche (Jacob, } \\
\text { et al., 2011). } \\
\text { Un enfant imité par un instructeur estime que ce dernier était plus attentif et l'a mieux } \\
\text { compris (Martin et Guéguen, 2015). } \\
\text { Une femme qui imite un homme en situation de speed-dating est jugé plus attrayante } \\
\text { sexuellement (Guéguen, 2009) }\end{array}$ \\
\hline Aide à autrui & $\begin{array}{l}\text { Une serveuse imitant verbalement un client lors de la prise de commande en répétant } \\
\text { littéralement sa commande reçoit par le suite plus de pourboires (Jacob \& Guéguen, 2013; } \\
\text { Van Baaren et al., 2003) } \\
\text { Une personne qui a imité quelqu'un auparavant et qui laisse tomber des choses par terre } \\
\text { sera plus aidé (Van Baaren et al., 2004). } \\
\text { Après avoir été imité par quelqu'un nous aidons plus quelqu'un d'autre qui ne nous a pas } \\
\text { imité et qui fait tomber des choses par terre (Van Baaren et al., 2004) ou qui nous demande } \\
\text { de l'argent dans la rue (Fischer-Lokou et al., 2011). }\end{array}$ \\
\hline
\end{tabular}




\begin{tabular}{|c|c|}
\hline & $\begin{array}{l}\text { Une personne qui a imité et qui sollicite ensuite un don au profit d'une association } \\
\text { caritative reçoit plus d'argent (Van Baaren et al., 2004, Kulesza et al, 2013). } \\
\text { Un étudiant consent plus favorablement à relire un mémoire d'un étudiant qu'il ne connaît } \\
\text { pas et qui l'a imité avant de faire sa requête (Guéguen, Martin, \& Meineri, 2011) }\end{array}$ \\
\hline Révélation sur soi & $\begin{array}{l}\text { Un enquêteur imitant la personne interviewée lors d'une enquête sur les pratiques sexuelles } \\
\text { conduira cette personne à faire des révélations plus intimes (Guéguen et al., 2013). } \\
\text { Un enquêteur qui imite la personne interviewée lors d'une enquête sur les comportements } \\
\text { de tris des déchets conduit la personne à donner des réponses plus honnêtes (Guéguen, } \\
2013 \text { ). } \\
\text { Un enfant imité par un instructeur confiera plus de bêtises qu'il a faites et de secrets } \\
\text { (Martin, } 2010 \text {; Martin et Guéguen, 2015). }\end{array}$ \\
\hline Comportement de consommation & $\begin{array}{l}\text { Une personne imitée par un vendeur/vendeuse achètera plus de produits proposés par } \\
\text { l'employé (Jacob, et al., 2011; Kulesza, et al., 2014). } \\
\text { On imite plus le comportement de consommation alimentaire d'une personne qui vient de } \\
\text { nous imiter (Johnston, 2002). } \\
\text { Parmi deux produits alimentaires, on choisira plus un produit lui-même retenu par une } \\
\text { personne qui vient de nous imiter (Tanner et al., 2008). } \\
\text { Un enfant acceptera plus de consommer des fruits et légumes qu'un instructeur lui } \\
\text { demande de consommer si il est imité au moment d'être interrogé sur sa consommation de } \\
\text { fruits et légumes (Martin, et al., in press) }\end{array}$ \\
\hline Persuasion & $\begin{array}{l}\text { Un avatar qui nous imite tout en diffusant un message sera jugé plus persuasif (Baileson et } \\
\text { Yee, 2005). On trouve le même effet lorsque l'on interagit avec un individu ayant des }\end{array}$ \\
\hline
\end{tabular}




\begin{tabular}{|c|c|}
\hline & attitudes non similaires aux nôtres et qui nous imite (Van Swol, 2003). \\
\hline Motivation et performances & $\begin{array}{l}\text { Un enfant imité par un instructeur qui lui demande de lire un texte suivi d'un rappel des } \\
\text { informations sur le test lira plus longtemps ce texte et aura des scores de rappel supérieurs } \\
\text { (Martin, 2010; Martin et Guéguen, 2015). } \\
\text { Les individus dont les caractéristiques les associent à des aptitudes et compétences moins } \\
\text { élevées confirme ce stéréotype (exemple femme moins bonne en maths que les hommes) } \\
\text { dans une tache de performance lorsqu'elles ont été imitées (Leander, Chartrand \& Wood, } \\
\text { 2011). }\end{array}$ \\
\hline Désir d'affiliation & $\begin{array}{l}\text { En situation de speed dating nous avons plus de désir de revoir la personne de sexe } \\
\text { opposée qui nous a imité (Guéguen, 2009; Guéguen, \& Martin, 2008) }\end{array}$ \\
\hline Négociation & $\begin{array}{l}\text { En situation de négociation, une personne imitée s'approchera plus de la proposition faite } \\
\text { par l'individu qui l'imite (Fischer et al., 2014; Maddux, Mullen \& Galinsky, 2007; Van } \\
\text { Swol, 2003). }\end{array}$ \\
\hline Jugement sur soi & $\begin{array}{l}\text { Un individu imité exprime par la suite plus de conscience de soi public et privé et moins } \\
\text { d'anxiété sociale (Guéguen, 2011). } \\
\text { Le fait d'être imité renforce l'estime de soi (Ashton-James, et al. } 2007 \text {; Kouzakova et al., } \\
\text { 2010) }\end{array}$ \\
\hline Cognitif & $\begin{array}{l}\text { Le fait d'être imité par nous conduit plus à avoir un style de traitement cognitif plus } \\
\text { dépendant du contexte (Van Baaren et al., 2004). } \\
\text { Un enfant imité par un instructeur se rappelle de plus d'informations sur un texte et } \\
\text { commet moins d'erreurs de rappel (Martin, 2010; Martin et Guéguen, 2015). } \\
\text { Des individus imités se révèlent plus performants à une tâche de Stroop et manifestent une }\end{array}$ \\
\hline
\end{tabular}




\begin{tabular}{|l|l|}
\hline Humeur et bien-être & meilleure fluidité verbale (Kouzakova, 2009). . \\
\hline & $\begin{array}{l}\text { Un enfant imité par un instructeur lors d'un entretien suivi d'une tache d'apprentissage } \\
\text { déclare plus de plaisir à avoir participé et déclare s'être senti plus à l'aise (Martin, 2010; } \\
\text { Martin et Guéguen, 2015) } \\
\text { Un individu imité exprime par la suite moins d'anxiété sociale (Guéguen, 2011). }\end{array}$ \\
\hline
\end{tabular}


Tableau 2: Tableau de synthèse des principales variables nous conduisant à imiter-pas imiter autrui

\begin{tabular}{|l|l|}
\hline Registre d'influence & Effets constatés et références \\
\hline $\begin{array}{l}\text { Appartenance de groupe (in group/out } \\
\text { group) }\end{array}$ & $\begin{array}{l}\text { On imite plus un compère qui se touche le visage lorsque l'on sait qu'il appartient au même } \\
\text { groupe religieux que nous (Yabar et al., 2006). } \\
\text { Une personne exclue dans un premier temps d'un groupe imitera plus une autre personne } \\
\text { qu'elle rencontre immédiatement après (Lakin, Chartrand, \& Arkin, 2008) } \\
\text { Une personne qui a pour instruction d'imiter des individus d'un autre groupe ethnique } \\
\text { jugera plus positivement ces derniers (Inzlicht, Gutsell, Legault, 2012). }\end{array}$ \\
\hline Désir de plaire & $\begin{array}{l}\text { On imite plus une personne attrayante de sexe opposé lorsque nous-mêmes sommes libres } \\
\text { de toute attache sentimentale (Farley, 2014; Karremans, \& Verwijmeren, 2008) }\end{array}$ \\
\hline Désir d'affiliation/Non affiliation & $\begin{array}{l}\text { Un amorçage avec des concepts reliés à l'affiliation (ensemble, ami...) conduit les } \\
\text { individus à plus imiter autrui par la suite (Lakin \& Chartrand, 2003). } \\
\text { Un amorçage avec des concepts liés à l'indépendance (je, moi, le mien...) conduit par la } \\
\text { suite les individus à moins imiter autrui (Van Baaren et al., 2003). }\end{array}$ \\
\hline Culpabilité & $\begin{array}{l}\text { Après une induction de culpabilité faisant croire à un participant qu'il a fait tomber les } \\
\text { affaires de quelqu'un d'autre on observe que le participant imite mieux un second compère } \\
\text { (Martin, Guéguen \& Fischer-Lokou, 2010) }\end{array}$ \\
\hline Statut & $\begin{array}{l}\text { Dans un groupe, on imite plus la personne qui a manifestement la position de leader } \\
\text { (Moore, 1995). } \\
\text { Lorsqu'un individu se retrouve dans une tâche à deux en position de subalterne par rapport }\end{array}$ \\
\hline
\end{tabular}




\begin{tabular}{|l|l|}
\hline Personnalité & $\begin{array}{l}\text { à un compère en position de leader, il imite plus ce dernier que quand les rôles sont } \\
\text { inversés (Cheng \& Chartrand, 2003). }\end{array}$ \\
\hline $\begin{array}{l}\text { Les individus ayant un haut niveau d'empathie et de préoccupation pour autrui imitent plus } \\
\text { une cible (Chartrand \& Bargh, 1999). } \\
\text { Les personnes ayant une forte dépendance au contexte imitent plus autrui (Van Barren et } \\
\text { al., 2004) }\end{array}$ \\
$\begin{array}{l}\text { Les individus avec un haut score de contrôle de soi imitent plus autrui (Cheng \& } \\
\text { Chartrand, 2003; Estow, Jamieson, Yates, 2007). } \\
\text { Les individus avec une personnalité narcissique imitent plus des individus mais } \\
\text { uniquement si le statut de ces individus est élevé (Ashton-James \& Levordashka, 2013) }\end{array}$ \\
\hline Humeur à autrui & $\begin{array}{l}\text { Des individus amorcés par des concepts en lien avec les comportements pro-sociaux } \\
\text { (coopérer, ensemble, équipe...) manifestent par la suite plus d'imitation (Leigthon et al., } \\
2010) .\end{array}$ \\
\hline $\begin{array}{l}\text { L'activation d'une humeur positive chez un individu le conduit par la suite à plus imiter } \\
\text { (Van Baaren, et al. 2006) }\end{array}$ \\
\hline
\end{tabular}


Tableau 3: Tableau de synthèse des principaux effets de l'imitation lorsque l'individu est conduit à inhiber/pas inhiber son comportement d'imitation

\begin{tabular}{|l|l|}
\hline Registre d'influence & Effets constatés et références \\
\hline Décodage des émotions & $\begin{array}{l}\text { L'impossibilité d'imiter conduit à accroître le temps mis pour décoder la nature d'une } \\
\text { émotion exprimée sur un visage et augmente les erreurs de décodage (Stel \& van } \\
\text { Knippenberg, 2008). } \\
\text { Une personne qui a pour instruction de ne pas imiter une cible est plus compétente pour } \\
\text { repérer un individu qui ment tandis qu'une personne qui a pour instruction d'imiter } \\
\text { apparaît moins performante (Stel, van Dijk, \& Olivier, 2009). }\end{array}$ \\
\hline Jugement d'autrui & $\begin{array}{l}\text { Les performances pour diagnostiquer un vrai/faux sourire diminuent chez des individus } \\
\text { ayant pour instruction de ne pas imiter (Rychlowska et al., 2014). }\end{array}$ \\
\hline Jugement sur soi & $\begin{array}{l}\text { Une instruction de non imitation des mouvements d'une cible conduit à moins l'apprécier } \\
\text { qu'une instruction d'imitation mais seulement lorsque cette cible est présentée } \\
\text { positivement (Stel et al., 2010). }\end{array}$ \\
\hline $\begin{array}{l}\text { Une personne qui a eu pour instruction d'imiter une cible se perçoit par la suite comme } \\
\text { plus concernée par les autres en général tandis que si elle a eu pour instruction de ne pas } \\
\text { imiter elle se perçoit comme moins concernée par autrui (Redeker, Stel \& Mastop, 2011) }\end{array}$ \\
\hline
\end{tabular}

